\title{
Cryptocurrency Returns before and after the Introduction of Bitcoin Futures
}

\author{
Pinar Deniz ${ }^{1, *(1)}$ and Thanasis Stengos ${ }^{2}$ \\ 1 Department of Economics, Marmara University, Istanbul 34722, Turkey \\ 2 Department of Economics, University of Guelph, Guelph, ON N1G 2W1, Canada; tstengos@uoguelph.ca \\ * Correspondence: pinar.deniz@marmara.edu.tr
}

Received: 7 May 2020; Accepted: 30 May 2020; Published: 4 June 2020

\begin{abstract}
This paper examines the behaviour of Bitcoin returns and those of several other cryptocurrencies in the pre and post period of the introduction of the Bitcoin futures market. We use the principal component-guided sparse regression (PC-LASSO) model to analyze several sample sizes for the pre and post periods. Besides the neighbourhood of the break time, the current period is also investigated as returns start to recover after some time. Search intensity is observed to be the most important variable for Bitcoin for all periods, whereas for the other cryptocurrencies there are other variables that seem more important in the pre period, while search intensity still stands out in the post period. Furthermore, GARCH analyses suggest that search intensity increases the volatility of Bitcoin returns more in the post period than it does in the pre period. Our empirical findings suggest that the top five cryptocurrencies are substitutes before the launch of Bitcoin futures. However, this effect is lost, and moreover, there are spillover effects on altcoins during both the post and the recovery period. We find a spillover effect of the introduction of bitcoin futures on altcoins and this effect seems to persist during the recovery period.
\end{abstract}

Keywords: bitcoin; cryptocurrencies; PC-LASSO; GARCH

JEL Classification: C38; G12; C15

\section{Introduction}

Cryptocurrencies have recently received attention both by the investors and technology-lovers due to several reasons such as: (i) they facilitate transactions by lowering costs and time-spending due to their peer-to-peer structure, i.e., excluding intermediaries and rendering the cryptocurrency-holder the governor of the account; (ii) they provide privacy in transactions which may matter for those who worry about account theft; (iii) they present relatively higher returns to the investors due to their volatile structure; (iv) they are not controlled by a central authority like other financial assets which provides an alternative for novelty-seekers; (v) they are popular and have already started to take part in the daily business and financial news network; (vi) last but not least, they have started to be used as a medium of exchange, such that you can buy yourself a cup of coffee with your coins.

The first digital currency, Bitcoin (BTC), created by the anonymous identity Satoshi Nakamoto (2008), is still dominating other cryptocurrencies ${ }^{1}$ by market capitalization. Reaching a market capitalization of over 170 billion US Dollars ${ }^{2}$, the futures market for Bitcoin was introduced by

1 There are currently more than 2300 currencies worldwide suggested by coinmarketcap.com as of the date of this study, 18 August 2019. Bitcoin is followed by Ethereum (ETH), Ripple (XRP), Bitcoin Cash (BHC) and Litecoin (LTC), in order, according to market capitalization.

2 It is constituting more than $65 \%$ of the total market capitalization of 250 billion US \$, as of 18 August 2019. 
10 December $2017^{3}$ to provide more transparency, liquidity and efficiency to the system. Including ups and downs, the Bitcoin price had seen an exponential rise until its drop-down in December 2017, which coincided with the introduction of the futures market (Corbet et al. 2018; Hale et al. 2018), even though this may be the result of an expected behaviour of a newly-established asset due to speculations (Stein 1987).

Recently, a vast empirical literature on cryptocurrencies has emerged. Some studies concentrate on the volatility of Bitcoin using GARCH-type models (Aalborg et al. 2019; Ardia et al. 2019; Bouoiyour and Selmi 2016; Bouri et al. 2017a; Charles and Darné 2019; Dyhrberg 2016a; Katsiampa 2017; Wu et al. 2019). Some studies analyze the volatility dynamics between different cryptocurrencies (Katsiampa 2018 , 2019). There are studies that examine the possibility of hedging cryptocurrencies with other assets (Dyhrberg 2016b; Bouri et al. 2017a, 2017b; Corbet et al. 2018; Pal and Mitra 2019; Wu et al. 2019; Sebastião and Godinho 2019). Some studies investigate the behaviour of Bitcoin whether it is a speculative investment or a currency (Aalborg et al. 2019; Bouoiyour and Selmi 2015; Corbet et al. 2018; Yermack 2015). In the literature, regarding their impact on cryptocurrencies, several macroeconomic and financial variables are investigated such as: search intensity (Dastgir et al. 2019; Kristoufek 2013; Panagiotidis et al. 2019); gold prices (Dyhrberg 2016a, 2016b; Wu et al. 2019); and (policy) uncertainty (Bouri et al. 2017b; Demir et al. 2018; Panagiotidis et al. 2019; Wu et al. 2019).

In this paper, we concentrate on the introduction of Bitcoin futures and use this date as the break point for pre and post analyses to examine the determinants of the top five cryptocurrencies, namely as, Bitcoin (BTC), Ethereum (ETH), Ripple (XRP), Bitcoin Cash (BHC) and Litecoin (LTC). Regarding the effect of the introduction of Bitcoin futures, Corbet et al. (2018), Liu et al. (2019) and Kim et al. (2019) observe a rise in the volatility, however the latter further added the information of a decline over time. Köchling et al. (2018) observe that introduction of futures market contributes only to Bitcoin in informational efficiency.

We use a principal component-guided sparse regression (PC-LASSO) model of Tay et al. (2018) for the identification of the most important variable. In the literature for cryptocurrencies, LASSO based models are employed by Panagiotidis et al. (2018), observing that search intensity, gold returns and policy uncertainty are the most important variables for Bitcoin returns; and by Panagiotidis et al. (2020), observing that policy uncertainty and stock market volatility are among the most important variables out of forty-one variables for the identification of Bitcoin returns using several subperiods of the overall period of 2010-2018. In this study, we use PC-LASSO to identify the most important variables regarding the returns of top five cryptocurrencies for the pre and post break point. We employ 12 subperiods to investigate the behaviour during the pre and post periods using samples of $30,60,90$, 120, 150 and 180 days. Empirical findings suggest that in the post break point period, search intensity is observed to be the most important variable out of several financial/macroeconomic variables for all cryptocurrencies examined. For the pre break point period, the most important variable is changing between different cryptocurrencies. Moreover, taking into account the non-normality of variables, the GARCH methodology is used to examine the behaviour of five cryptocurrencies for the pre and post break point period with the main drivers included as exogenous variables in the model. Empirical findings are consistent regarding the impact of search intensity on returns for the post break point period for all cryptocurrencies. Finally, the recovery period (Bitcoin prices started to recover after December 2018) is investigated to get information about the recent behaviour of the cryptocurrencies, which again highlights search intensity as the most important variable.

The rest of the paper is organized as follows. Section 2 describes data and methodologies of PC-LASSO and six different GARCH models. Section 3 discusses the empirical findings. Section 4 concludes the paper.

3 This is the listing date of the first Bitcoin futures market by the Chicago Board Options Exchange (CBOE). However, CBOE has announced to stop listing Bitcoin futures by March 2019. Chicago Mercantile Exchange (CME) has launched Bitcoin futures market by 10 December 2017. 


\section{Data and Methodology}

\subsection{Data}

The dataset ${ }^{4}$ consists of 4 cryptocurrencies other than Bitcoin ${ }^{5}$, namely as, Ethereum, Ripple, Bitcoin Cash and Litecoin, and several financial or macroeconomic variables as their potential determinants, namely as, gold prices (Gold), NYSE index (NYSE), oil price, Google search intensity (GTrends), China economic policy uncertainty (China EPU), VIX index (VIX), federal funds rate (FFR) and US economic policy uncertainty (US EPU). December 10, 2017 is selected as the threshold date (the first trade date for Bitcoin futures) and 30, 60, 90, 120, 150 and 180 days before and after this date is used as the time span ${ }^{6}$. The datasets are obtained from different sources given in Table 1 . All datasets, except for the Federal Funds Rate (FFR), are daily returns. The figures for daily closing prices and price returns are given in Figures A1 and A2 with the break time of the introduction of futures for Bitcoin depicted in dashed line. Descriptive statistics highlight the non-normality of all variables, given in Table 2.

Table 1. Dataset.

\begin{tabular}{|c|c|c|c|c|}
\hline Variable & Source & Code & Frequency & Type \\
\hline Cryptocurrencies & coinmarketcap.com & - & daily & $\%$ change \\
\hline$€ / \$$ & quandl.com & FRED/DEXUSEU & daily & $\%$ change \\
\hline Gold & quandl.com & WGC/GOLD_DAILY_USD & daily & $\%$ change \\
\hline NYSE & quandl.com & $\mathrm{BCB} / 7809$ & daily & $\%$ change \\
\hline Oil price & quandl.com & FRED/DCOILBRENTEU & daily & $\%$ change \\
\hline GTrends & "gtrendsR" R package & - & daily & $\%$ change \\
\hline China EPU & policyuncertainty.com & - & monthly & $\%$ change \\
\hline VIX & cboe.com & - & daily & $\%$ change \\
\hline FFR & quandl.com & FED/RIFSPFF_N_D & daily & levels \\
\hline US EPU & policyuncertainty.com & - & daily & $\%$ change \\
\hline
\end{tabular}

Note: Cryptocurrencies include all top 5 cryptocurrencies' returns using daily close prices. $e / \$$ is U.S./Euro foreign exchange rate. Gold is gold prices in US dollars. NYSE is Dow Jones NYSE index. Oil price is in US dollars per barrel for crude oil prices for Brent - Europe. GTrends is an index between 0-100 to measure the number of searches for the term "bitcoin" in Google. China EPU index is linearly interpolated to daily frequency. VIX is Chicago Board Options Exchange (CBOE) volatility index in US dollars. FFR is daily federal funds effective rate. US EPU is the economic policy uncertainty index for the US.

Table 2. Summary statistics.

\begin{tabular}{lccccccc}
\hline & BTC & $\boldsymbol{e} / \mathbf{\$}$ & Gold & NYSE & Oil Price & GTrends & China EPU \\
\hline Mean & 0.47 & 0.00 & 0.01 & 0.05 & 0.14 & 1.51 & 0.21 \\
Max. & 25.25 & 0.98 & 2.59 & 2.33 & 4.64 & 100.00 & 2.58 \\
Min. & -18.74 & -1.06 & -1.88 & -4.15 & -4.32 & -38.00 & -3.70 \\
Std. Dev. & 5.44 & 0.31 & 0.46 & 0.59 & 1.13 & 18.84 & 1.26 \\
Skewness & 0.24 & 0.01 & 0.58 & -1.48 & 0.00 & 1.73 & -1.09 \\
Kurtosis & 5.10 & 4.80 & 8.25 & 14.81 & 5.32 & 9.34 & 4.25 \\
JB & $61.63^{* *}$ & $43.24^{* *}$ & $384.98^{* *}$ & $1971.16^{* *}$ & $71.84^{* *}$ & $693.08^{* *}$ & $83.55^{* *}$ \\
\hline & VIX & FFR & US EPU & ETH & XRP & CASH & LTC \\
\hline Mean & 0.20 & 1.37 & 16.48 & 0.49 & 0.81 & 0.88 & 0.61 \\
Max. & 115.60 & 1.70 & 564.19 & 26.46 & 83.47 & 53.97 & 47.60 \\
Min. & -22.87 & 1.06 & -79.58 & -22.81 & -29.76 & -35.98 & -32.64 \\
Std. Dev. & 9.35 & 0.22 & 74.56 & 5.99 & 9.83 & 11.05 & 7.81 \\
Skewness & 6.52 & 0.41 & 2.61 & 0.10 & 3.21 & 1.41 & 1.51 \\
Kurtosis & 76.83 & 1.69 & 14.55 & 5.19 & 24.48 & 8.36 & 11.47 \\
JB & $74,722.37^{* *}$ & $31.90^{* *}$ & $2134.85^{* *}$ & $64.48^{* *}$ & $6677.14 * *$ & $486.74^{* *}$ & $1074.70 * *$ \\
\hline
\end{tabular}

Note: JB is Jarque-Bera statistics with the null hypothesis of normality. $\left.{ }^{* *}\right)$ denotes significance at $5 \%$. All variables except for FFR are in percentage changes.

4 The dataset is available on demand.

5 Top 5 cryptocurrencies are selected according to their market capitalization as of 18 August 2019.

$6 \quad 13$ June 2017 to 7 June 2018 is the 360 days of the overall time span. 


\subsection{Methodology}

\subsubsection{PC-LASSO}

In order to identify the most important variables that determine the returns of the most traded cryptocurrencies, we apply principal component-guided sparse regression (PC-LASSO, Tay et al. 2018) following Panagiotidis et al. (2020). This is a new method for large data to shrink predictions toward the most important principle components of the feature matrix by combining LASSO sparsity penalty with a quadratic penalty. Hence, the method is a combination of LASSO and principle components regression. This technique solves the optimization problem below for $\ell(y, \eta)$, negative log-likelihood function for observation using the observation weight, $\omega$ :

$$
\min _{\beta_{0}, \beta} \frac{1}{n} \sum_{i=1}^{N} w_{i} \ell\left(y_{i}, \beta_{0}+\beta^{T} x_{i}\right)+\lambda\|\beta\|_{1}+\frac{\theta}{2} \sum_{k=1}^{K} \beta_{k}^{T} V_{k} D_{d_{k 1}^{2}-d_{k j}^{2}} V_{k}^{T} \beta_{k} .
$$

where $n$ is the number of observations, $x_{i}$ is feature vector for each observation and $y_{i}$, the response. The data features may come in $K$ groups or $K$ may simply be equal to one, referring to no group. $U_{k} D_{k} V_{k}^{T}$ is singular value decomposition for each feature matrix and $D_{d_{k 1}^{2}-d_{k j}^{2}}$ is the diagonal matrix. The multiplier for the quadratic penalty is presented by $\theta$.

In this study, PC-LASSO test is implemented using R package of "pcLasso", taking the observation weight equal to 1 for each observation, the ratio of shrinkage equal to 0.8 and the features belong to a single group.

\subsubsection{GARCH-M Model}

The six GARCH models used in this paper consist of an autoregressive term $\left(y_{t-1}\right)$, the exogenous variable $\left(X_{t}\right)$ and volatility term $\left(\sigma_{t}\right)$ in the conditional mean equation, which renders them GARCH in mean (GARCH-M) models. The exogenous variable is selected as the most important variable observed from the PC-LASSO method for pre and post periods, i.e., $X_{t}^{\text {pre }}$ and $X_{t}^{\text {post }}$, according to the introduction of futures ${ }^{7}$. Each conditional variance equation also includes these, given in Equation (2).

Conditional mean equation for the basic GARCH model:

$$
y_{t}=\lambda_{0}+\lambda_{1} y_{t-1}+\lambda_{2} \sigma_{t}+\kappa^{\text {pre }} X_{t}^{\text {pre }}+\kappa^{\text {post }} X_{t}^{\text {post }}+\epsilon_{t}
$$

Conditional variance equation for the basic GARCH model:

$$
\sigma_{t}^{2}=\omega+\beta \sigma_{t-1}^{2}+\alpha \epsilon_{t-1}^{2}+\mu^{\text {pre }} X_{t}^{\text {pre }}+\mu^{\text {post }} X_{t}^{\text {post }}
$$

Apart from the basic GARCH model (Bollerslev 1986), we employ five different GARCH-type models, namely as, TGARCH (Glosten et al. 1993), EGARCH (Nelson 1991), Asymmetric Power ARCH (APARCH, (Ding et al. 1993)), Component GARCH (CGARCH) and Asymmetric Component GARCH (ACGARCH) (Engle and Lee 1993) models. The model with the highest log-likelihood (LL) will be selected as the best model out of 6 different GARCH specifications.

7 Hence, $X_{t}=X_{t}^{\text {pre }}+X_{t}^{\text {post }}$. 


\section{Empirical Findings}

All variables except for FFR are in percentage changes and are stationary ${ }^{8}$. In order to be able to compare the coefficients obtained from empirical analyses, all variables are standardized to have a mean of 0 and variance of 1 .

Table 3 presents the PC-LASSO coefficients for each sample for Bitcoin. For each time period, Google search intensity is observed to be the most important as it provides the highest coefficient out of nine potential drivers, in line with Bouoiyour and Selmi (2015) and Panagiotidis et al. (2018). The coefficients of search intensity are positive for each pre-futures period and negative for each post-futures period. This finding signifies the date of the launch of Bitcoin futures as a break point consistent with the literature (Akyildirim et al. 2019; Liu et al. 2019).

Tables A1-A4 presents PC-LASSO coefficients for Ethereum, Ripple, Bitcoin Cash and Litecoin, successively. The findings for Ethereum highlight the importance of (i) search intensity in the pre-futures period for the samples between 30 and 60 days whereas it is $€ / \$$ for the samples of 90 days and more; (ii) search intensity in the post-futures period for the samples of 60 days and more. $€ / \$$ is observed to have a positive effect on Ethereum returns for the pre-futures period whereas search intensity has a negative effect for the post-futures period similar to Bitcoin. The findings for Ripple highlight the importance of (i) US EPU in the pre-futures period for the samples between 30 and 90 days whereas it is VIX for the samples of 120 and more; (ii) search intensity in the post-futures period for the samples of 60 days and more. Similar to Bitcoin, search intensity has a negative effect on Ripple returns for post-futures period, whereas US EPU has a positive and VIX has a negative effect for the pre-futures period. The findings for Bitcoin Cash highlight the importance of (i) gold in the pre-futures period for the sample of 30 days whereas it is NYSE for the samples between 60 and 120 days; (ii) search intensity in the post-futures period for the samples of 60 days and more. Similar to Bitcoin, search intensity is negatively affecting Bitcoin Cash returns for the post-futures period. In the pre-futures period, NYSE also has a negative effect. Lastly, the findings for Litecoin highlight the importance of (i) search intensity in the pre-futures period for the samples of 30 days and the samples of 90 days and more; (ii) FFR in the post-futures period for the samples between 30 and 90 days whereas it is search intensity for the samples of 120 days and more. Similar to Bitcoin, search intensity is negatively affecting Litecoin returns for post-futures period. FFR is also observed to have a negative effect on returns for the post-futures period for samples closer to the chosen break point. For the pre-futures period, search intensity is also found to have an overall negative effect.

The skewness and kurtosis statistics and Jarque-Bera test shown in Table 2 suggest the absence of normality for all variables. Following Katsiampa (2017) and Charles and Darné (2019), we estimate six GARCH models for all cryptocurrency returns under the existence of an exogenous variable (it is incorporated in two separate forms as pre and post-futures period), which is search intensity for Bitcoin and Litecoin as it stands out as the main driver from PC-LASSO, whereas for other cryptocurrencies, the most important variables in the pre-futures period are varying, i.e., $€ / \$$, VIX and NYSE for Ethereum, Ripple and Bitcoin Cash, respectively.

8 Unit root test results are available on demand. 
Table 3. Principal component-guided sparse regression (PC-LASSO) coefficients for Bitcoin.

\begin{tabular}{lcccccc}
\hline Variable & Pre-30 & Post-30 & Pre-60 & Post-60 & Pre-90 & Post-90 \\
\hline$€ / \$$ & 0.00000 & -0.02872 & 0.00000 & 0.00000 & 0.00000 & 0.00000 \\
Gold & -0.00165 & 0.00000 & 0.00000 & 0.00000 & 0.00000 & 0.00000 \\
NYSE & 0.00000 & 0.05370 & 0.00000 & 0.02231 & 0.00000 & 0.00000 \\
Oil price & -0.00669 & 0.00000 & 0.00000 & 0.00000 & -0.08133 & 0.00000 \\
GTrends & $\mathbf{0 . 0 8 2 1 1}$ & $-\mathbf{0 . 0 7 2 5 2}$ & $\mathbf{0 . 1 4 0 2 0}$ & $-\mathbf{0 . 1 4 1 6 1}$ & $\mathbf{0 . 1 3 0 5 2}$ & $-\mathbf{0 . 1 3 0 8 3}$ \\
China & 0.00000 & 0.00000 & 0.00000 & 0.00000 & 0.00000 & 0.00000 \\
VIX & 0.00000 & -0.03311 & 0.00000 & -0.06211 & 0.00000 & -0.05824 \\
FFR & 0.00000 & 0.00000 & 0.00000 & 0.00000 & 0.00000 & 0.00000 \\
EPU & 0.00000 & 0.04333 & 0.00000 & 0.00000 & 0.00000 & 0.00000 \\
\hline Variable & $\mathbf{P r e - 1 2 0}$ & Post-120 & Pre-150 & Post-150 & Pre-180 & Post-180 \\
\hline$€ / \$$ & 0.00000 & 0.00000 & 0.01970 & 0.00000 & 0.00139 & 0.00000 \\
Gold & 0.00000 & 0.00000 & 0.00000 & 0.00000 & 0.00000 & 0.00000 \\
NYSE & 0.00000 & 0.00000 & 0.00000 & 0.00000 & 0.00000 & 0.00000 \\
Oil price & -0.03595 & 0.00000 & -0.01879 & 0.00000 & 0.00000 & 0.00000 \\
GTrends & $\mathbf{0 . 1 3 6 1 3}$ & $-\mathbf{0 . 1 0 8 8 1}$ & $\mathbf{0 . 0 9 5 0 1}$ & $-\mathbf{0 . 0 8 9 5 7}$ & $\mathbf{0 . 0 8 5 1 0}$ & $-\mathbf{0 . 0 9 4 9 0}$ \\
China & 0.00000 & 0.00000 & 0.00000 & 0.00000 & 0.00000 & 0.00000 \\
VIX & 0.00000 & -0.04241 & 0.00000 & -0.04599 & 0.00000 & -0.03787 \\
FFR & 0.00000 & 0.00000 & 0.00000 & 0.00000 & 0.00000 & 0.00000 \\
EPU & 0.00000 & 0.00000 & 0.00000 & 0.00000 & 0.00000 & 0.00000 \\
\hline
\end{tabular}

Note: Each sample is divided into two periods at the date 10-Dec-2017. Sample 60: 10-Nov-2017 to 8-Jan-2018; sample 120: 11-Oct-2017 to 7-Feb-2018; sample 180: 11-Sep-2017 to 9-Mar-2018; sample 240: 12-Aug-2017 to 8-Apr-2018; sample 300: 13-Jul-2017 to 8-May-2018; sample 360: 13-Jun-2017 to 7-Jun-2018.

Table 4 reflects the results for all GARCH models for Bitcoin returns for the largest sample size of 360 days. $\mathrm{ARCH}(5), Q^{2}(10)$ and JB are diagnostic test results for heteroscedasticity, serial correlation and normality for standardized residuals, successively. Empirical findings suggest APARCH as the best model according to log-likelihood and also information criteria. Diagnostics for the APARCH model are also appropriate since the hypotheses of no heteroscedasticity and no serial correlation have not failed or been rejected. The finding of negative impact of search intensity on returns for the post-future period is consistent with PC-LASSO findings. Moreover, search intensity is observed to be positively affecting volatility for both periods and the impact during pre-futures period is higher compared to the post-futures period. Last but not least, volatility is found to have a positive impact on Bitcoin returns, as observed in the mean equation.

Tables A5-A8 suggest that search intensity has a negative effect on returns for the post-future period for all other cryptocurrencies, as well. These results are in line with PC-LASSO findings for altcoins. Moreover, search intensity is observed to be positively affecting the conditional volatilities of altcoins except for Bitcoin Cash.

As an additional robustness analysis, a recovery period (the period is selected informally by eyeballing from 15 December 2018 up to the latest available (8 August 2019)) is checked to investigate the recent behaviours of cryptocurrency returns. Table A9 presents PC-LASSO coefficients for the relevant period. Search intensity is again observed to be the main driver not only for Bitcoin returns but also for all other cryptocurrency returns. Tables A10-A14 provides GARCH results for the recovery period. As an exogenous variable, search intensity is incorporated both into the conditional mean and the variance equations for all cryptocurrencies during the recovery period. GARCH tests reflect that search intensity is increasing the conditional volatilities of all cryptocurrencies including Bitcoin, whereas the impact on returns is strictly positive only for Bitcoin. 
Table 4. GARCH-M Tests for Bitcoin for the sample of 360 days.

\begin{tabular}{|c|c|c|c|c|c|c|}
\hline & GARCH & TGARCH & EGARCH & APARCH & CGARCH & ACGARCH \\
\hline \multicolumn{7}{|l|}{ Mean Equation } \\
\hline$\lambda_{0}$ & $\begin{array}{c}-0.3130 * \\
(0.1679)\end{array}$ & $\begin{array}{l}-0.2056 \\
(0.2005)\end{array}$ & $\begin{array}{l}-0.3532 \\
(0.6283)\end{array}$ & $\begin{array}{c}-0.3742 * * \\
(0.1508)\end{array}$ & $\begin{array}{l}-0.0416 \\
(0.1240)\end{array}$ & $\begin{array}{c}-0.4107^{* * * *} \\
(0.1140)\end{array}$ \\
\hline$\lambda_{1}$ & $\begin{array}{l}-0.0685 \\
(0.0624)\end{array}$ & $\begin{array}{l}-0.0722 \\
(0.0559)\end{array}$ & $\begin{array}{l}-0.0674 \\
(0.0431)\end{array}$ & $\begin{array}{l}-0.0499 \\
(0.0562)\end{array}$ & $\begin{array}{l}-0.0440 \\
(0.0550)\end{array}$ & $\begin{array}{l}-0.0802 \\
(0.0513)\end{array}$ \\
\hline$\lambda_{2}$ & $\begin{array}{l}0.3772 * \\
(0.2065)\end{array}$ & $\begin{array}{c}0.2207 \\
(0.2474)\end{array}$ & $\begin{array}{c}0.3676 \\
(0.6946)\end{array}$ & $\begin{array}{c}0.4128^{* *} \\
(0.1998)\end{array}$ & $\begin{array}{c}0.0489 \\
(0.1583)\end{array}$ & $\begin{array}{c}0.4921^{* * *} \\
(0.1692)\end{array}$ \\
\hline$\kappa_{\text {pre }}$ & $\begin{array}{c}0.0949 \\
(0.0992)\end{array}$ & $\begin{array}{c}0.1363 \\
(0.0954)\end{array}$ & $\begin{array}{c}0.0263 \\
(0.1860)\end{array}$ & $\begin{array}{c}0.0854 \\
(0.0955)\end{array}$ & $\begin{array}{c}0.1892 * * * \\
(0.0610)\end{array}$ & $\begin{array}{l}0.1227^{*} \\
(0.0656)\end{array}$ \\
\hline$\kappa_{\text {post }}$ & $\begin{array}{c}-0.3078^{* * *} \\
(0.0765)\end{array}$ & $\begin{array}{c}-0.2884^{* * * *} \\
(0.0781)\end{array}$ & $\begin{array}{c}-0.3625^{* * *} \\
(0.1238)\end{array}$ & $\begin{array}{c}-0.3137^{* * *} \\
(0.0962)\end{array}$ & $\begin{array}{c}-0.3172 * * * \\
(0.0809)\end{array}$ & $\begin{array}{c}-0.3621^{* * * *} \\
(0.0690)\end{array}$ \\
\hline \multicolumn{7}{|l|}{ Variance Equation } \\
\hline$\omega$ & $\begin{array}{c}0.2285^{* * *} \\
(0.0663)\end{array}$ & $\begin{array}{l}0.2020^{* * *} \\
(0.0552)\end{array}$ & $\begin{array}{c}-0.3394^{* *} \\
(0.1612)\end{array}$ & $\begin{array}{c}0.1320 * * * \\
(0.0414)\end{array}$ & $\begin{array}{c}0.8275^{* * *} \\
(0.1541)\end{array}$ & $\begin{array}{c}0.6977^{* * *} \\
(0.1206)\end{array}$ \\
\hline $\mathrm{ARCH}(\alpha)$ & $\begin{array}{l}0.1172 * * \\
(0.0467)\end{array}$ & $\begin{array}{c}0.0547 \\
(0.0413)\end{array}$ & $\begin{array}{c}0.0962 \\
(0.1447)\end{array}$ & $\begin{array}{c}0.0816^{* * *} \\
(0.0232)\end{array}$ & $\begin{array}{c}0.2421 \\
(0.7781)\end{array}$ & $\begin{array}{c}0.1115 \\
(0.2192)\end{array}$ \\
\hline GARCH $(\beta)$ & $\begin{array}{c}0.6147^{* * *} \\
(0.0917)\end{array}$ & $\begin{array}{c}0.6530 * * * \\
(0.0872)\end{array}$ & $\begin{array}{l}-0.1126 \\
(0.1403)\end{array}$ & $\begin{array}{c}0.8061^{* * *} \\
(0.0526)\end{array}$ & $\begin{array}{c}0.6998 \\
(0.7025)\end{array}$ & $\begin{array}{l}0.7331^{* *} \\
(0.3045)\end{array}$ \\
\hline$\mu_{\text {pre }}$ & $\begin{array}{c}0.4204^{* * *} \\
(0.0827)\end{array}$ & $\begin{array}{c}0.4145^{* * *} \\
(0.0547)\end{array}$ & $\begin{array}{c}0.6586^{* * *} \\
(0.1612)\end{array}$ & $\begin{array}{c}0.1482 * * * \\
(0.0256)\end{array}$ & $\begin{array}{c}0.1995^{* * *} \\
(0.0424)\end{array}$ & $\begin{array}{c}0.2764^{* * *} \\
(0.1016)\end{array}$ \\
\hline$\mu_{\text {post }}$ & $\begin{array}{c}0.1136 \\
(0.0703)\end{array}$ & $\begin{array}{l}0.1314^{*} \\
(0.0767)\end{array}$ & $\begin{array}{l}0.3069^{* *} \\
(0.1553)\end{array}$ & $\begin{array}{l}0.0561 * \\
(0.0315)\end{array}$ & $\begin{array}{c}0.2625^{* * *} \\
(0.0884)\end{array}$ & $\begin{array}{c}0.0811 \\
(0.0931)\end{array}$ \\
\hline TGARCH $(\gamma)$ & & $\begin{array}{c}0.0914 \\
(0.0715)\end{array}$ & & & & \\
\hline $\operatorname{EGARCH}(\delta)$ & & & $\begin{array}{l}-0.0804 \\
(0.0952)\end{array}$ & & & \\
\hline $\operatorname{APARCH}(\gamma)$ & & & & $\begin{array}{c}0.9594^{* * *} \\
(0.1210)\end{array}$ & & \\
\hline (A)CGARCH $(\rho)$ & & & & & $\begin{array}{c}0.9601 * * * \\
(0.0199)\end{array}$ & $\begin{array}{c}0.7914^{* * *} \\
(0.1124)\end{array}$ \\
\hline (A)CGARCH $(\theta)$ & & & & & $\begin{array}{l}-0.1511 \\
(0.7997)\end{array}$ & $\begin{array}{l}-0.0841 \\
(0.2323)\end{array}$ \\
\hline $\operatorname{ACGARCH}(\gamma)$ & & & & & & $\begin{array}{c}0.0703 \\
(0.0705)\end{array}$ \\
\hline AIC & 2.5983 & 2.5829 & 2.6158 & 2.5616 & 2.5732 & 2.5839 \\
\hline SIC & 2.7065 & 2.7019 & 2.7456 & 2.6806 & 2.7138 & 2.7353 \\
\hline LL & -456.40 & -452.63 & -457.53 & -448.82 & -448.88 & -449.81 \\
\hline JB stat & $\begin{array}{l}16.5278 \\
{[0.0003]}\end{array}$ & $\begin{array}{l}12.4547 \\
{[0.0020]}\end{array}$ & $\begin{array}{l}16.9623 \\
{[0.0002]}\end{array}$ & $\begin{array}{l}10.3891 \\
{[0.0055]}\end{array}$ & $\begin{array}{l}10.7820 \\
{[0.0046]}\end{array}$ & $\begin{array}{l}15.6510 \\
{[0.0004]}\end{array}$ \\
\hline $\mathrm{ARCH}(5)$ & $\begin{array}{c}1.4018 \\
{[0.2229]}\end{array}$ & $\begin{array}{c}1.5379 \\
{[0.1773]}\end{array}$ & $\begin{array}{c}4.3657 \\
{[0.0007]}\end{array}$ & $\begin{array}{c}0.7529 \\
{[0.5844]}\end{array}$ & $\begin{array}{c}1.4881 \\
{[0.1930]}\end{array}$ & $\begin{array}{c}0.7208 \\
{[0.6082]}\end{array}$ \\
\hline$Q^{2}(10)$ & $\begin{array}{c}4.3556 \\
{[0.9300]}\end{array}$ & $\begin{array}{c}4.6183 \\
{[0.9150]}\end{array}$ & $\begin{array}{c}7.9512 \\
{[0.6340]}\end{array}$ & $\begin{array}{c}2.5770 \\
{[0.9900]}\end{array}$ & $\begin{array}{c}3.3294 \\
{[0.9730]}\end{array}$ & $\begin{array}{c}5.0435 \\
{[0.8880]}\end{array}$ \\
\hline
\end{tabular}

Notes: $(*),(* *)$ and $(* * *)$ denote significance at $10 \%, 5 \%$ and $1 \%$, successively. Both for pre and post-futures period, search intensity is used as the exogeneous variable, according to PC-LASSO findings. Values in paranthesis are standard deviations. Values in brackets are $p$-values for the diagnostic test. Sample covers daily period between 13-Jun-17 and 7-Jun-18. AIC is Akaike Information Criterion, SIC is Schwarz Information Criterion, LL is Log-likelihood. There are 6 GARCH models used:GARCH: $\sigma_{t}^{2}=\omega+\alpha \epsilon_{t-1}^{2}+\beta \sigma_{t-1}^{2}+\mu^{\text {pre }} X_{t}^{\text {pre }}+\mu^{\text {post }} X_{t}^{\text {post }}$. TGARCH: $\sigma_{t}^{2}=\omega+\alpha \epsilon_{t-1}^{2}+\beta \sigma_{t-1}^{2}-\gamma \epsilon_{t-1}^{2} I_{t-1}+\mu^{\text {post }} X_{t}^{\text {post }}$. EGARCH: $\log \left(\sigma_{t}^{2}\right)=\omega+\alpha\left(\frac{\left|\epsilon_{t-1}\right|}{\left|\sigma_{t-1}\right|}-\sqrt{2 / \pi}\right)+$ $\beta \log \left(\sigma_{t-1}^{2}\right)+\delta \frac{\left|\epsilon_{t-1}\right|}{\left|\sigma_{t-1}\right|}+\mu^{\text {post }} X_{t}^{\text {post }}$. APARCH: $\sigma_{t}^{\delta}=\omega+\alpha\left(\left|\epsilon_{t-1}\right|-\gamma \epsilon_{t-1}\right)^{\delta}+\beta \sigma_{t-1}^{\delta}+\mu^{\text {post }} X_{t}^{\text {post }}$. CGARCH: $\sigma_{t}^{2}=q_{t}+\alpha\left(\epsilon_{t-1}^{2}-q_{t-1}\right)+\beta\left(\sigma_{t-1}^{2}-q_{t-1}\right)+\mu^{\text {post }} X_{t}^{\text {post }} . q_{t}=\omega+\rho\left(q_{t-1}-\omega\right)+\theta\left(\epsilon_{t-1}^{2}-\sigma_{t-1}^{2}\right)$. ACGARCH: $\sigma_{t}^{2}=q_{t}+\alpha\left(\epsilon_{t-1}^{2}-q_{t-1}\right)+\gamma\left(\epsilon_{t-1}^{2}-q_{t-1}\right) D_{t-1}+\beta\left(\sigma_{t-1}^{2}-q_{t-1}\right)+\mu^{\text {post }} X_{t}^{\text {post }} \cdot q_{t}=\omega+\rho\left(q_{t-1}-\omega\right)+\theta\left(\epsilon_{t-1}^{2}-\sigma_{t-1}^{2}\right)$. 


\section{Conclusions}

This study investigates the behaviour of cryptocurrencies before and after the launch of Bitcoin futures market by 10 December 2017. Several potential drivers of cryptocurrency returns are investigated using the PC-LASSO test for different sample sizes around the neighborhood of the launch date. The results suggest Google search intensity as the most important variable for Bitcoin returns for all samples. However, the sign of the effect is changing depending on the pre and post-periods. Search intensity is observed to have a positive impact on Bitcoin returns during pre period, whereas it is negative during the post period. For other cryptocurrency returns, search intensity is generally observed to be the main driver during the post-futures period. However, the main driver is varying depending on the sample and the cryptocurrency during the pre-futures period, yet during the post-futures period, they are mainly affected by the same indicator, i.e., search intensity. Hence, we may interpret this finding as suggesting that altcoins are substitutes for Bitcoin before the introduction of the futures market; however, this result may not hold during the post-futures period.

Moreover, volatilities are examined so as to further investigate the behaviour of the cryptocurrencies. Several GARCH models are tested using the largest sample size of 360 days with the exogeneous variables determined by PC-LASSO. The effect of post-futures search intensity is observed to have a negative impact on all cryptocurrency returns, which is in line with PC-LASSO findings. According to the GARCH models, search intensity is increasing the volatility of cryptocurrency returns except for Bitcoin Cash.

As a further analysis, for the recovery period, the PC-LASSO results suggest that the same indicator, search intensity, is the most important and has an effect in the same direction, something that may be interpreted to imply that these cryptocurrencies are no longer substitutes. Last but not least, the main driver, search intensity, incorporated into the GARCH models, is observed to be increasing conditional volatilities of all cryptocurrencies, whereas the impact on conditional returns is positive for Bitcoin but is not effective for the rest of the altcoins. To conclude, our empirical findings suggest that the top five cryptocurrencies are substitutes before the launch of Bitcoin futures. However, this effect is lost and, moreover, there are spillover effects on altcoins during both the post and the recovery period. As a policy recommendation, it may be suggested that holding different cryptocurrencies may not be an optimal risk diversification policy as the most traded cryptocurrencies tend to be mainly affected by the same indicator and in the same direction.

Author Contributions: P.D. and T.S. have contributed to each section equally. All authors have read and agreed to the published version of the manuscript.

Funding: This research received no external funding.

Conflicts of Interest: The authors declare no conflict of interest.

\section{Appendix A. Pre and Post Period Analyses}

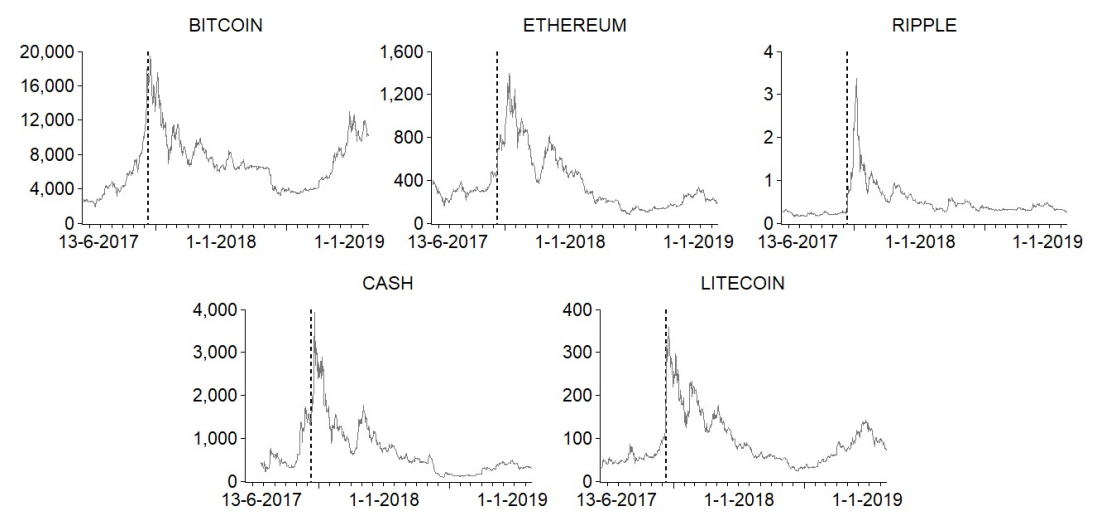

Figure A1. Cryptocurrency prices. 

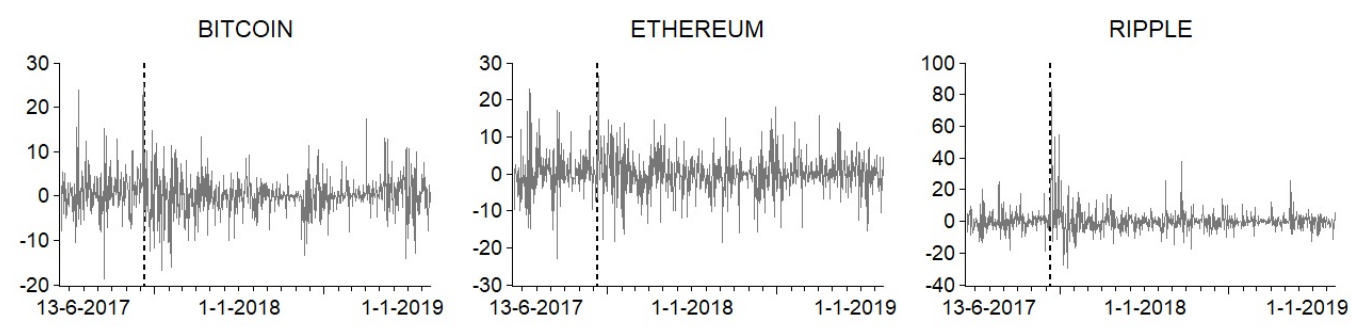

$\mathrm{CASH}$

LITECOIN
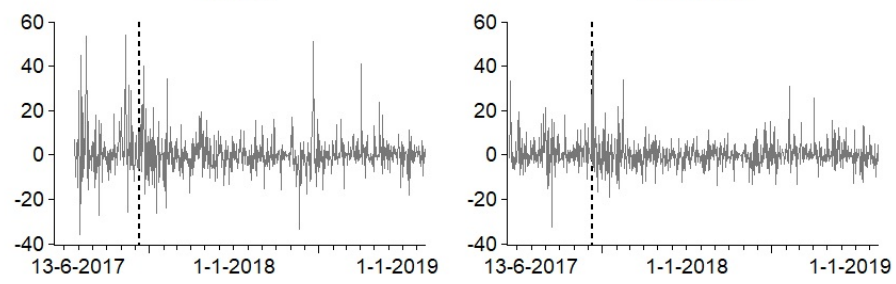

Figure A2. Cryptocurrency returns.

Table A1. PC-LASSO coefficients for ethereum.

\begin{tabular}{lcccccc}
\hline Variable & Pre-30 & Post-30 & Pre-60 & Post-60 & Pre-90 & Post-90 \\
\hline$€ / \$$ & 0.05797 & -0.06112 & 0.04370 & 0.00000 & $\mathbf{0 . 0 8 5 2 8}$ & 0.00000 \\
Gold & 0.01325 & 0.00000 & 0.03587 & 0.00000 & 0.00127 & 0.00000 \\
NYSE & 0.00000 & 0.00686 & 0.00000 & 0.03944 & 0.00000 & 0.00000 \\
Oil price & 0.00000 & -0.02636 & 0.02719 & 0.00000 & -0.01326 & 0.00000 \\
GTrends & $-\mathbf{0 . 0 8 5 9 2}$ & -0.07312 & $-\mathbf{0 . 0 9 0 3 0}$ & $-\mathbf{0 . 1 4 1 2 4}$ & -0.03892 & $-\mathbf{0 . 1 4 0 6 7}$ \\
China & 0.00000 & -0.00051 & 0.00000 & 0.00000 & 0.00000 & 0.00000 \\
VIX & 0.00000 & 0.00000 & -0.03018 & -0.04220 & -0.01026 & -0.03466 \\
FFR & 0.00000 & $-\mathbf{0 . 0 8 2 6 7}$ & 0.00000 & -0.04378 & 0.00000 & -0.05206 \\
EPU & 0.00000 & 0.00000 & 0.00000 & 0.00000 & 0.00000 & 0.00000 \\
\hline Variable & $\mathbf{P r e - 1 2 0}$ & $\mathbf{P o s t - 1 2 0}$ & $\mathbf{P r e - 1 5 0}$ & $\mathbf{P o s t - 1 5 0}$ & Pre-180 & Post-180 \\
\hline €/\$ & $\mathbf{0 . 0 7 2 1 4}$ & 0.00000 & $\mathbf{0 . 0 7 6 8 6}$ & 0.00000 & $\mathbf{0 . 0 5 8 7 3}$ & 0.00000 \\
Gold & 0.02406 & 0.00000 & 0.00000 & 0.00000 & 0.01647 & 0.00000 \\
NYSE & 0.00000 & 0.00000 & 0.00000 & 0.00000 & 0.00000 & 0.00000 \\
Oil price & 0.00000 & 0.00000 & -0.01884 & 0.00000 & 0.00000 & 0.00000 \\
GTrends & -0.04826 & $-\mathbf{0 . 1 1 3 9 4}$ & 0.00000 & $-\mathbf{0 . 0 9 4 2 5}$ & 0.00000 & $-\mathbf{0 . 0 9 5 7 1}$ \\
China & 0.00000 & 0.00000 & 0.00000 & 0.00000 & 0.03256 & 0.00000 \\
VIX & -0.01647 & -0.02691 & 0.00000 & -0.03041 & 0.00000 & -0.02155 \\
FFR & 0.00000 & -0.01705 & 0.00000 & 0.00000 & 0.00000 & 0.00000 \\
EPU & 0.00000 & 0.00000 & 0.00000 & 0.00000 & 0.00000 & 0.00000 \\
\hline
\end{tabular}

Notes: Same notes as in Table 3.

Table A2. PC-LASSO coefficients for Ripple.

\begin{tabular}{lcccccc}
\hline Variable & Pre-30 & Post-30 & Pre-60 & Post-60 & Pre-90 & Post-90 \\
\hline €/\$ & 0.00000 & 0.00000 & 0.00000 & 0.00000 & 0.00000 & 0.00000 \\
Gold & 0.00000 & 0.00000 & 0.06074 & 0.00565 & 0.05274 & 0.00000 \\
NYSE & 0.02333 & 0.00000 & 0.00000 & 0.00000 & 0.01320 & 0.00000 \\
Oil price & 0.00292 & 0.00000 & 0.00000 & 0.00000 & 0.00000 & 0.00000 \\
GTrends & -0.07065 & -0.05535 & -0.06001 & $-\mathbf{0 . 0 9 9 5 6}$ & -0.06491 & $-\mathbf{0 . 1 0 6 5 0}$ \\
China & 0.00000 & 0.02429 & 0.00000 & 0.00000 & 0.00000 & 0.00000 \\
VIX & 0.00000 & $\mathbf{0 . 0 9 0 3 1}$ & -0.01531 & 0.00000 & -0.04728 & -0.08191 \\
FFR & 0.00000 & -0.01027 & 0.00000 & -0.07337 & 0.00000 & 0.00000 \\
EPU & $\mathbf{0 . 0 8 2 9 8}$ & 0.00000 & $\mathbf{0 . 1 1 1 8 1}$ & 0.00000 & $\mathbf{0 . 0 8 6 4 4}$ & 0.00000 \\
\hline
\end{tabular}


Table A2. Cont.

\begin{tabular}{lcccccc}
\hline Variable & Pre-120 & Post-120 & Pre-150 & Post-150 & Pre-180 & Post-180 \\
\hline$€ / \$$ & 0.00000 & 0.00000 & 0.00000 & 0.00000 & 0.00000 & 0.00000 \\
Gold & 0.00000 & 0.00000 & 0.00000 & 0.00000 & 0.00000 & 0.00000 \\
NYSE & 0.05410 & 0.00000 & 0.02893 & 0.00000 & 0.04384 & 0.00000 \\
Oil price & 0.00000 & 0.00000 & 0.00000 & 0.00000 & 0.00000 & 0.00000 \\
GTrends & -0.00497 & $-\mathbf{0 . 0 8 5 9 8}$ & -0.01832 & $-\mathbf{0 . 0 7 4 2 1}$ & -0.02516 & $-\mathbf{0 . 0 7 7 8 8}$ \\
China & 0.00000 & 0.00000 & 0.00000 & 0.00000 & 0.01425 & 0.00000 \\
VIX & $-\mathbf{0 . 0 9 0 6 9}$ & 0.00000 & $-\mathbf{0 . 0 5 7 6 1}$ & 0.00000 & $-\mathbf{0 . 0 4 9 4 9}$ & 0.00000 \\
FFR & 0.00000 & -0.03492 & -0.00740 & -0.00225 & -0.01483 & -0.00834 \\
EPU & 0.02202 & 0.00000 & 0.02258 & 0.00000 & 0.02380 & 0.00000 \\
\hline
\end{tabular}

Notes: Same notes as in Table 3.

Table A3. PC-LASSO coefficients for Bitcoin cash.

\begin{tabular}{lcccccc}
\hline Variable & Pre-30 & Post-30 & Pre-60 & Post-60 & Pre-90 & Post-90 \\
\hline €/\$ & 0.00000 & 0.02043 & 0.00000 & 0.00000 & 0.00000 & 0.00000 \\
Gold & $\mathbf{0 . 0 6 6 3 6}$ & 0.00000 & 0.00762 & 0.00782 & 0.01986 & 0.00000 \\
NYSE & -0.02661 & $-\mathbf{0 . 0 6 7 3 9}$ & $-\mathbf{0 . 0 5 9 7 8}$ & 0.00000 & $-\mathbf{0 . 0 5 3 3 5}$ & 0.00000 \\
Oil price & 0.00000 & -0.00465 & 0.00529 & 0.00000 & 0.00000 & 0.00000 \\
GTrends & 0.00000 & 0.00000 & 0.00000 & $-\mathbf{0 . 0 9 7 9 4}$ & 0.00000 & $-\mathbf{0 . 1 0 8 7 8}$ \\
China & 0.00000 & 0.00128 & -0.01140 & 0.00000 & -0.03693 & 0.00000 \\
VIX & 0.02349 & 0.02554 & 0.00924 & -0.05607 & 0.00989 & -0.00845 \\
FFR & 0.00000 & 0.00000 & 0.00000 & 0.00000 & 0.00000 & 0.00000 \\
EPU & 0.00000 & 0.00000 & 0.00000 & 0.00000 & -0.02171 & 0.00000 \\
\hline Variable & Pre-120 & Post-120 & Pre-150 & Post-150 & Pre-180 & Post-180 \\
\hline €/\$ & 0.00000 & 0.00000 & & 0.00000 & & 0.00000 \\
Gold & 0.01163 & 0.00000 & & 0.00000 & & 0.00000 \\
NYSE & $-\mathbf{0 . 0 9 2 9 9}$ & 0.00000 & & 0.00000 & & 0.00000 \\
Oil price & 0.00000 & 0.00000 & & 0.00000 & & 0.00000 \\
GTrends & 0.00000 & $-\mathbf{0 . 0 8 8 2 5}$ & & $-\mathbf{0 . 0 7 6 8 8}$ & & $-\mathbf{0 . 0 8 2 3 5}$ \\
China & 0.00000 & 0.00000 & & 0.00000 & & 0.00000 \\
VIX & 0.02653 & -0.00629 & & -0.01039 & & -0.00046 \\
FFR & 0.00000 & 0.00000 & & 0.00000 & & 0.00000 \\
EPU & 0.00000 & 0.00000 & & 0.00000 & & 0.00000
\end{tabular}

Notes: Results for pre-150 and pre-180 are not available since Bitcoin Cash began trading by 1 August 2017.

Table A4. PC-LASSO coefficients for Litecoin.

\begin{tabular}{lcccccc}
\hline Variable & Pre-30 & Post-30 & Pre-60 & Post-60 & Pre-90 & Post-90 \\
\hline €/\$ & 0.00000 & -0.11001 & 0.00000 & -0.01104 & 0.00428 & 0.00000 \\
Gold & 0.00000 & 0.00000 & 0.00000 & 0.00000 & 0.00674 & 0.00000 \\
NYSE & 0.00000 & 0.00000 & 0.00000 & 0.00000 & 0.00000 & 0.00000 \\
Oil price & 0.02470 & -0.00478 & 0.02981 & 0.00000 & -0.01220 & 0.00000 \\
GTrends & $-\mathbf{0 . 0 9 0 5 1}$ & 0.00000 & -0.07668 & -0.08981 & $-\mathbf{0 . 0 5 8 5 7}$ & -0.08395 \\
China & 0.02133 & 0.00000 & $\mathbf{0 . 0 8 8 6 4}$ & 0.00000 & 0.00000 & 0.00000 \\
VIX & 0.00000 & 0.00000 & 0.00000 & -0.00148 & -0.02796 & -0.04056 \\
FFR & 0.00000 & $-\mathbf{0 . 1 1 5 8 1}$ & 0.00000 & $-\mathbf{0 . 1 3 0 5 3}$ & 0.00000 & $-\mathbf{0 . 1 2 2 5 8}$ \\
EPU & 0.00000 & 0.00000 & 0.00000 & 0.00000 & 0.00000 & 0.00000 \\
\hline Variable & Pre-120 & Post-120 & Pre-150 & Post-150 & Pre-180 & Post-180 \\
\hline €/\$ & 0.00000 & 0.00000 & 0.00877 & 0.00000 & 0.00000 & 0.00000 \\
Gold & 0.04201 & 0.00000 & 0.01465 & 0.00000 & 0.00328 & 0.00000 \\
NYSE & 0.00000 & 0.01919 & 0.00000 & 0.01539 & 0.00000 & 0.00803 \\
Oil price & 0.00000 & 0.00000 & 0.00000 & 0.00000 & 0.00000 & 0.00000 \\
GTrends & $-\mathbf{0 . 0 5 4 9 6}$ & $-\mathbf{0 . 0 8 1 9 5}$ & $-\mathbf{0 . 0 4 0 4 9}$ & $-\mathbf{0 . 0 7 0 4 9}$ & $-\mathbf{0 . 0 4 5 1 6}$ & $-\mathbf{0 . 0 7 3 9 8}$ \\
China & 0.00000 & 0.00000 & 0.00441 & 0.00000 & 0.00000 & 0.00000 \\
VIX & -0.03459 & -0.04667 & -0.03398 & -0.04767 & -0.03155 & -0.04121 \\
FFR & 0.00000 & -0.05128 & -0.00172 & -0.00206 & 0.00000 & -0.00627 \\
EPU & 0.00000 & 0.00000 & 0.00747 & 0.00000 & 0.00000 & 0.00000 \\
\hline
\end{tabular}

Notes: Same notes as in Table 3. 
Table A5. GARCH-M Tests for Ethereum for the sample of 360 days.

\begin{tabular}{|c|c|c|c|c|c|c|}
\hline & GARCH & TGARCH & EGARCH & APARCH & CGARCH & ACGARCH \\
\hline \multicolumn{7}{|l|}{ Mean Equation } \\
\hline$\lambda_{0}$ & $\begin{array}{c}-0.4675 * * * \\
(0.1506)\end{array}$ & $\begin{array}{l}-0.1141 \\
(0.2416)\end{array}$ & $\begin{array}{c}-0.7912 * \\
(0.4619)\end{array}$ & $\begin{array}{c}-0.4358^{* * *} \\
(0.1685)\end{array}$ & $\begin{array}{c}-0.2475 * \\
(0.1450)\end{array}$ & $\begin{array}{c}-0.7289 * * * * \\
(0.2204)\end{array}$ \\
\hline$\lambda_{1}$ & $\begin{array}{l}-0.0350 \\
(0.0499)\end{array}$ & $\begin{array}{l}-0.0010 \\
(0.0572)\end{array}$ & $\begin{array}{c}-0.1585^{* * *} \\
(0.0601)\end{array}$ & $\begin{array}{c}0.0251 \\
(0.0489)\end{array}$ & $\begin{array}{c}-0.0992 \text { ** } \\
(0.0491)\end{array}$ & $\begin{array}{c}-0.1089 * * \\
(0.0525)\end{array}$ \\
\hline$\lambda_{2}$ & $\begin{array}{c}0.5264^{* * * *} \\
(0.1727)\end{array}$ & $\begin{array}{c}0.1282 \\
(0.2738)\end{array}$ & $\begin{array}{c}0.7816 \\
(0.5049)\end{array}$ & $\begin{array}{c}0.4585 * * \\
(0.2032)\end{array}$ & $\begin{array}{c}0.2081 \\
(0.1660)\end{array}$ & $\begin{array}{c}0.7805^{* * * *} \\
(0.2510)\end{array}$ \\
\hline$\kappa_{\text {pre }}$ & $\begin{array}{c}0.0555 \\
(0.0891)\end{array}$ & $\begin{array}{c}0.0856 \\
(0.0825)\end{array}$ & $\begin{array}{c}0.0417 \\
(0.0796)\end{array}$ & $\begin{array}{c}0.0980 * * \\
(0.0415)\end{array}$ & $\begin{array}{c}0.0488 \\
(0.0575)\end{array}$ & $\begin{array}{c}0.0396 \\
(0.0669)\end{array}$ \\
\hline$\kappa_{\text {post }}$ & $\begin{array}{c}-0.3571^{* * *} \\
(0.0719)\end{array}$ & $\begin{array}{c}-0.2048^{* * *} \\
(0.0761)\end{array}$ & $\begin{array}{c}-0.3597^{* * * *} \\
(0.1195)\end{array}$ & $\begin{array}{c}-0.2482^{* * *} \\
(0.0719)\end{array}$ & $\begin{array}{c}-0.2984^{* * *} \\
(0.0689)\end{array}$ & $\begin{array}{c}-0.4509^{* * *} \\
(0.0735)\end{array}$ \\
\hline \multicolumn{7}{|l|}{ Variance Equation } \\
\hline$\omega$ & $\begin{array}{c}0.7580 * * * \\
(0.0680)\end{array}$ & $\begin{array}{c}0.1202^{* *} \\
(0.0472)\end{array}$ & $\begin{array}{c}-0.4421^{* *} \\
(0.1838)\end{array}$ & $\begin{array}{c}0.1502 * * * \\
(0.0039)\end{array}$ & $\begin{array}{c}1.0254^{* * *} \\
(0.2319)\end{array}$ & $\begin{array}{c}0.9304^{* * * *} \\
(0.1406)\end{array}$ \\
\hline $\mathrm{ARCH}(\alpha)$ & $\begin{array}{c}0.3373^{* * *} \\
(0.0772)\end{array}$ & $\begin{array}{c}0.0819 * * \\
(0.0327)\end{array}$ & $\begin{array}{c}0.3985^{* *} \\
(0.1862)\end{array}$ & $\begin{array}{c}0.1696^{* * *} \\
(0.0324)\end{array}$ & $\begin{array}{c}0.2683 * * * \\
(0.0939)\end{array}$ & $\begin{array}{c}0.0889 \\
(0.0679)\end{array}$ \\
\hline $\operatorname{GARCH}(\beta)$ & $\begin{array}{c}-0.1310 * * * \\
(0.0461)\end{array}$ & $\begin{array}{c}0.7165^{* * *} \\
(0.0795)\end{array}$ & $\begin{array}{l}-0.0340 \\
(0.1689)\end{array}$ & $\begin{array}{c}0.7091^{* * *} \\
(0.0240)\end{array}$ & $\begin{array}{l}-0.2371 \\
(0.1661)\end{array}$ & $\begin{array}{c}0.1826 \\
(0.1130)\end{array}$ \\
\hline$\mu_{\text {pre }}$ & $\begin{array}{c}0.0566 \\
(0.1007)\end{array}$ & $\begin{array}{l}-0.0515 \\
(0.0609)\end{array}$ & $\begin{array}{c}0.1132 \\
(0.1550)\end{array}$ & $\begin{array}{l}-0.0196 \\
(0.0182)\end{array}$ & $\begin{array}{c}0.0629 \\
(0.0635)\end{array}$ & $\begin{array}{c}0.0142 \\
(0.0512)\end{array}$ \\
\hline$\mu_{\text {post }}$ & $\begin{array}{c}0.2796^{* * *} \\
(0.0511)\end{array}$ & $\begin{array}{c}0.2581^{* * *} \\
(0.0645)\end{array}$ & $\begin{array}{l}0.3316^{*} \\
(0.1930)\end{array}$ & $\begin{array}{c}0.0888^{* * *} \\
(0.0302)\end{array}$ & $\begin{array}{c}0.3164^{* * *} \\
(0.1004)\end{array}$ & $\begin{array}{c}0.0753 \\
(0.1232)\end{array}$ \\
\hline TGARCH $(\gamma)$ & & $\begin{array}{l}0.1501 * \\
(0.0873)\end{array}$ & & & & \\
\hline EGARCH $(\delta)$ & & & $\begin{array}{c}0.0393 \\
(0.0979)\end{array}$ & & & \\
\hline \multicolumn{7}{|l|}{ Variance Equation } \\
\hline $\operatorname{APARCH}(\gamma)$ & & & & $\begin{array}{c}0.1035 \\
(0.1412)\end{array}$ & & \\
\hline (A)CGARCH $(\rho)$ & & & & & $\begin{array}{c}0.9480 * * * \\
(0.0323)\end{array}$ & $\begin{array}{c}0.9361^{* * *} \\
(0.0239)\end{array}$ \\
\hline (A)CGARCH $(\theta)$ & & & & & $\begin{array}{c}0.0368 \\
(0.0332)\end{array}$ & $\begin{array}{c}0.0057 \\
(0.0235)\end{array}$ \\
\hline ACGARCH $(\gamma)$ & & & & & & $\begin{array}{c}0.3570 * * * \\
(0.1112)\end{array}$ \\
\hline AIC & 2.6756 & 2.6935 & 2.6392 & 2.6934 & 2.5995 & 2.6234 \\
\hline SIC & 2.7838 & 2.8125 & 2.7690 & 2.8124 & 2.7401 & 2.7748 \\
\hline LL & -470.27 & -472.49 & -461.73 & -472.46 & -453.60 & -456.90 \\
\hline JB stat & $\begin{array}{c}33.30 \\
{[0.0000]}\end{array}$ & $\begin{array}{c}33.54 \\
{[0.0000]}\end{array}$ & $\begin{array}{c}38.92 \\
{[0.0000]}\end{array}$ & $\begin{array}{c}26.12 \\
{[0.0000]}\end{array}$ & $\begin{array}{c}43.18 \\
{[0.0000]}\end{array}$ & $\begin{array}{c}64.73 \\
{[0.0000]}\end{array}$ \\
\hline $\mathrm{ARCH}(5)$ & $\begin{array}{c}1.9968 \\
{[0.0785]}\end{array}$ & $\begin{array}{c}0.3147 \\
{[0.9040]}\end{array}$ & $\begin{array}{c}0.0286 \\
{[0.8660]}\end{array}$ & $\begin{array}{c}0.3058 \\
{[0.9093]}\end{array}$ & $\begin{array}{c}1.2229 \\
{[0.2978]}\end{array}$ & $\begin{array}{c}0.7757 \\
{[0.5678]}\end{array}$ \\
\hline$Q^{2}(10)$ & $\begin{array}{c}1.3503 \\
{[0.2450]}\end{array}$ & $\begin{array}{c}0.4346 \\
{[0.5100]}\end{array}$ & $\begin{array}{c}1.9568 \\
{[0.0845]}\end{array}$ & $\begin{array}{c}0.0000 \\
{[0.9950]}\end{array}$ & $\begin{array}{c}0.8557 \\
{[0.3550]}\end{array}$ & $\begin{array}{c}0.3332 \\
{[0.5640]}\end{array}$ \\
\hline
\end{tabular}

Notes: $\left.\left({ }^{*}\right),{ }^{* *}\right)$ and $\left({ }^{* * *}\right)$ denote significance at $10 \%, 5 \%$ and $1 \%$, successively. During the pre-futures period, $e / \$$ is used as the exogeneous variable whereas it is search intensity for the post-futures period, according to PC-LASSO findings. 
Table A6. GARCH-M Tests for Ripple for the sample of 360 days.

\begin{tabular}{|c|c|c|c|c|c|c|}
\hline & GARCH & TGARCH & EGARCH & APARCH & CGARCH & ACGARCH \\
\hline \multicolumn{7}{|l|}{ Mean Equation } \\
\hline$\lambda_{0}$ & $\begin{array}{l}-0.2030 \\
(0.1602)\end{array}$ & $\begin{array}{c}-0.3726^{* *} \\
(0.1896)\end{array}$ & $\begin{array}{c}-0.2401 * * * \\
(0.0798)\end{array}$ & $\begin{array}{c}-0.3679 * * * \\
(0.0911)\end{array}$ & $\begin{array}{c}-0.2793 * * * \\
(0.0770)\end{array}$ & $\begin{array}{c}-0.3668^{* * *} \\
(0.0968)\end{array}$ \\
\hline$\lambda_{1}$ & $\begin{array}{l}-0.0284 \\
(0.0641)\end{array}$ & $\begin{array}{l}-0.0520 \\
(0.0639)\end{array}$ & $\begin{array}{c}-0.0999 * * \\
(0.0413)\end{array}$ & $\begin{array}{c}-0.2151^{* * *} \\
(0.0400)\end{array}$ & $\begin{array}{c}-0.1066^{* *} \\
(0.0434)\end{array}$ & $\begin{array}{c}-0.1031 \text { ** } \\
(0.0440)\end{array}$ \\
\hline$\lambda_{2}$ & $\begin{array}{c}0.1545 \\
(0.2028)\end{array}$ & $\begin{array}{l}0.3915^{*} \\
(0.2380)\end{array}$ & $\begin{array}{c}0.1310 \\
(0.1067)\end{array}$ & $\begin{array}{c}0.4189 * * * \\
(0.1167)\end{array}$ & $\begin{array}{l}0.1809 * \\
(0.1089)\end{array}$ & $\begin{array}{c}0.3061 * * \\
(0.1267)\end{array}$ \\
\hline$\kappa_{\text {pre }}$ & $\begin{array}{l}-0.0228 \\
(0.0238)\end{array}$ & $\begin{array}{c}0.0050 \\
(0.0496)\end{array}$ & $\begin{array}{l}-0.0185 \\
(0.0257)\end{array}$ & $\begin{array}{l}-0.0092 \\
(0.0330)\end{array}$ & $\begin{array}{l}-0.0207 \\
(0.0233)\end{array}$ & $\begin{array}{l}-0.0276 \\
(0.0306)\end{array}$ \\
\hline$\kappa_{\text {post }}$ & $\begin{array}{c}-0.1985^{* *} \\
(0.0900)\end{array}$ & $\begin{array}{c}-0.2365^{* * *} \\
(0.0862)\end{array}$ & $\begin{array}{c}-0.1177^{* * *} \\
(0.0457)\end{array}$ & $\begin{array}{c}-0.3163^{* * *} \\
(0.0584)\end{array}$ & $\begin{array}{c}-0.1161 \text { ** } \\
(0.0453)\end{array}$ & $\begin{array}{c}-0.1662 \text { ** } \\
(0.0536)\end{array}$ \\
\hline \multicolumn{7}{|l|}{ Variance Equation } \\
\hline$\omega$ & $\begin{array}{l}0.0587^{* * *} \\
(0.0082)\end{array}$ & $\begin{array}{l}0.0840^{* * *} \\
(0.0118)\end{array}$ & $\begin{array}{c}-0.2302 * * * \\
(0.0583)\end{array}$ & $\begin{array}{c}0.2145^{* * *} \\
(0.0303)\end{array}$ & $\begin{array}{l}0.5443^{* * *} \\
(0.1832)\end{array}$ & $\begin{array}{c}0.5788^{* * *} \\
(0.2172)\end{array}$ \\
\hline $\mathrm{ARCH}(\alpha)$ & $\begin{array}{c}0.1516^{* * *} \\
(0.0226)\end{array}$ & $\begin{array}{c}0.2374 * * * \\
(0.0376)\end{array}$ & $\begin{array}{c}0.2668^{* * *} \\
(0.0816)\end{array}$ & $\begin{array}{c}-0.0512 * * * \\
(0.0080)\end{array}$ & $\begin{array}{c}0.2191 * * * \\
(0.0143)\end{array}$ & $\begin{array}{l}0.1181 * \\
(0.0661)\end{array}$ \\
\hline GARCH $(\beta)$ & $\begin{array}{c}0.7970 * * * \\
(0.0195)\end{array}$ & $\begin{array}{c}0.7493^{* * *} \\
(0.0245)\end{array}$ & $\begin{array}{c}0.9089^{* * *} \\
(0.0298)\end{array}$ & $\begin{array}{c}0.8111^{* * *} \\
(0.0328)\end{array}$ & $\begin{array}{l}-0.1932 \\
(0.1199)\end{array}$ & $\begin{array}{l}-0.0921 \\
(0.1309)\end{array}$ \\
\hline$\mu_{\text {pre }}$ & $\begin{array}{c}-0.0881^{* * *} \\
(0.0230)\end{array}$ & $\begin{array}{c}-0.0980^{* * *} \\
(0.0107)\end{array}$ & $\begin{array}{l}-0.2875^{*} \\
(0.1482)\end{array}$ & $\begin{array}{c}-0.1153^{* * *} \\
(0.0144)\end{array}$ & $\begin{array}{c}-0.0532 * * * \\
(0.0204)\end{array}$ & $\begin{array}{l}-0.0402 \\
(0.0327)\end{array}$ \\
\hline$\mu_{\text {post }}$ & $\begin{array}{c}0.2080 * * * \\
(0.0557)\end{array}$ & $\begin{array}{c}0.1444^{* *} \\
(0.0663)\end{array}$ & $\begin{array}{c}0.2364 \\
(0.1602)\end{array}$ & $\begin{array}{l}-0.0082 \\
(0.0154)\end{array}$ & $\begin{array}{c}0.1896^{* * *} \\
(0.0537)\end{array}$ & $\begin{array}{c}0.2470 * * * \\
(0.0764)\end{array}$ \\
\hline TGARCH $(\gamma)$ & & $\begin{array}{c}-0.1718^{* * *} \\
(0.0431)\end{array}$ & & & & \\
\hline EGARCH $(\delta)$ & & & $\begin{array}{c}0.0857 \\
(0.0550)\end{array}$ & & & \\
\hline $\operatorname{APARCH}(\gamma)$ & & & & $\begin{array}{c}0.9995^{* * *} \\
(0.0125)\end{array}$ & & \\
\hline \multicolumn{7}{|l|}{ Variance Equation } \\
\hline (A)CGARCH $(\rho)$ & & & & & $\begin{array}{c}0.9788^{* * *} \\
(0.0120)\end{array}$ & $\begin{array}{c}0.9864^{* * *} \\
(0.0082)\end{array}$ \\
\hline (A)CGARCH $(\theta)$ & & & & & $\begin{array}{c}0.0425^{* * *} \\
(0.0017)\end{array}$ & $\begin{array}{c}0.0204^{* * *} \\
(0.0078)\end{array}$ \\
\hline $\operatorname{ACGARCH}(\gamma)$ & & & & & & $\begin{array}{c}0.2784^{* * *} \\
(0.0693)\end{array}$ \\
\hline AIC & 2.5585 & 2.5409 & 2.3566 & 2.7027 & 2.3191 & 2.3491 \\
\hline SIC & 2.6666 & 2.6599 & 2.4864 & 2.8217 & 2.4597 & 2.5006 \\
\hline LL & -449.24 & -445.10 & -411.01 & -474.13 & -403.27 & -407.67 \\
\hline JB stat & $\begin{array}{c}432.02 \\
{[0.0000]}\end{array}$ & $\begin{array}{c}314.41 \\
{[0.0000]}\end{array}$ & $\begin{array}{c}511.96 \\
{[0.0000]}\end{array}$ & $\begin{array}{c}514.13 \\
{[0.0000]}\end{array}$ & $\begin{array}{c}703.90 \\
{[0.0000]}\end{array}$ & $\begin{array}{c}822.11 \\
{[0.0000]}\end{array}$ \\
\hline $\mathrm{ARCH}(5)$ & $\begin{array}{c}0.9767 \\
{[0.4319]}\end{array}$ & $\begin{array}{c}0.5925 \\
{[0.7057]}\end{array}$ & $\begin{array}{c}0.6531 \\
{[0.6594]}\end{array}$ & $\begin{array}{c}5.9086 \\
{[0.0000]}\end{array}$ & $\begin{array}{c}1.2792 \\
{[0.2723]}\end{array}$ & $\begin{array}{c}2.6045 \\
{[0.0249]}\end{array}$ \\
\hline$Q^{2}(10)$ & $\begin{array}{l}3.1287 \\
{[0.077]}\end{array}$ & $\begin{array}{c}1.6729 \\
{[0.1960]}\end{array}$ & $\begin{array}{c}1.5658 \\
{[0.2110]}\end{array}$ & $\begin{array}{l}18.7030 \\
{[0.0000]}\end{array}$ & $\begin{array}{c}0.1352 \\
{[0.7130]}\end{array}$ & $\begin{array}{c}0.899 \\
{[0.343]}\end{array}$ \\
\hline
\end{tabular}

Notes: $\left({ }^{*}\right),\left({ }^{* *}\right)$ and $\left({ }^{* * *}\right)$ denote significance at $10 \%, 5 \%$ and $1 \%$, successively. During the pre-futures period, VIX is used as the exogeneous variable whereas it is search intensity for the post-futures period, according to PC-LASSO findings. 
Table A7. GARCH-M Tests for Bitcoin Cash for the sample of 360 days.

\begin{tabular}{|c|c|c|c|c|c|c|}
\hline & GARCH & TGARCH & EGARCH & APARCH & CGARCH & ACGARCH \\
\hline \multicolumn{7}{|l|}{ Mean Equation } \\
\hline$\lambda_{0}$ & $\begin{array}{c}-0.6232 * * * \\
(0.2298)\end{array}$ & $\begin{array}{c}-1.0192 * * * \\
(0.1967)\end{array}$ & $\begin{array}{l}-0.4045^{* *} \\
(0.1723)\end{array}$ & $\begin{array}{c}-1.1033^{* * *} \\
(0.2288)\end{array}$ & $\begin{array}{c}-0.2813^{*} \\
(0.1500)\end{array}$ & $\begin{array}{c}-0.2343^{*} \\
(0.1366)\end{array}$ \\
\hline$\lambda_{1}$ & $\begin{array}{l}-0.1056 \\
(0.0729)\end{array}$ & $\begin{array}{l}-0.0959 \\
(0.0731)\end{array}$ & $\begin{array}{l}-0.0678 \\
(0.0547)\end{array}$ & $\begin{array}{c}-0.1104 \text { ** } \\
(0.0452)\end{array}$ & $\begin{array}{l}-0.0692 \\
(0.0531)\end{array}$ & $\begin{array}{l}-0.0482 \\
(0.0527)\end{array}$ \\
\hline$\lambda_{2}$ & $\begin{array}{c}0.6630 * * \\
(0.2739)\end{array}$ & $\begin{array}{c}1.1014 * * * \\
(0.2369)\end{array}$ & $\begin{array}{c}0.3151 \\
(0.2099)\end{array}$ & $\begin{array}{c}1.2664^{* * *} \\
(0.2646)\end{array}$ & $\begin{array}{c}0.1618 \\
(0.1801)\end{array}$ & $\begin{array}{c}0.1037 \\
(0.1631)\end{array}$ \\
\hline$\kappa_{\text {pre }}$ & $\begin{array}{l}0.0914 \text { * } \\
(0.0505)\end{array}$ & $\begin{array}{c}0.1226^{* *} \\
(0.0502)\end{array}$ & $\begin{array}{c}0.0521 \\
(0.0452)\end{array}$ & $\begin{array}{c}0.1622 * * * \\
(0.0534)\end{array}$ & $\begin{array}{c}0.0500 \\
(0.0423)\end{array}$ & $\begin{array}{c}0.0340 \\
(0.0455)\end{array}$ \\
\hline$\kappa_{\text {post }}$ & $\begin{array}{c}-0.4645^{* * *} \\
(0.0982)\end{array}$ & $\begin{array}{c}-0.4766^{* * *} \\
(0.0912)\end{array}$ & $\begin{array}{c}-0.4100^{* * *} \\
(0.0636)\end{array}$ & $\begin{array}{c}-0.3608^{* * *} \\
(0.0680)\end{array}$ & $\begin{array}{c}-0.4021^{* * *} \\
(0.0566)\end{array}$ & $\begin{array}{c}-0.3883^{* * * *} \\
(0.0560)\end{array}$ \\
\hline \multicolumn{7}{|l|}{ Variance Equation } \\
\hline$\omega$ & $\begin{array}{l}0.3829^{* * *} \\
(0.0762)\end{array}$ & $\begin{array}{l}0.5656^{* * *} \\
(0.0811)\end{array}$ & $\begin{array}{c}-0.4941^{* * *} \\
(0.1333)\end{array}$ & $\begin{array}{l}0.5197^{* * *} \\
(0.0654)\end{array}$ & $\begin{array}{c}0.9669^{* * *} \\
(0.3132)\end{array}$ & $\begin{array}{c}1.0479 * * * \\
(0.4068)\end{array}$ \\
\hline $\mathrm{ARCH}(\alpha)$ & $\begin{array}{c}0.3385^{* * *} \\
(0.0902)\end{array}$ & $\begin{array}{c}0.2685^{* * *} \\
(0.0781)\end{array}$ & $\begin{array}{c}0.5618^{* * *} \\
(0.1903)\end{array}$ & $\begin{array}{c}0.2139 * * * \\
(0.0318)\end{array}$ & $\begin{array}{l}0.2204 * \\
(0.1331)\end{array}$ & $\begin{array}{c}0.2430 \\
(0.1615)\end{array}$ \\
\hline GARCH $(\beta)$ & $\begin{array}{c}0.2665 * * \\
(0.1088)\end{array}$ & $\begin{array}{c}-0.0186^{* * *} \\
(0.0888)\end{array}$ & $\begin{array}{c}0.6070 * * * \\
(0.1368)\end{array}$ & $\begin{array}{c}0.2823^{* * *} \\
(0.0734)\end{array}$ & $\begin{array}{l}-0.0631 \\
(0.3172)\end{array}$ & $\begin{array}{l}-0.0331 \\
(0.3434)\end{array}$ \\
\hline$\mu_{\text {pre }}$ & $\begin{array}{c}-0.1618^{* * *} \\
(0.0320)\end{array}$ & $\begin{array}{c}-0.1902 * * * \\
(0.0223)\end{array}$ & $\begin{array}{c}-0.3808^{* * *} \\
(0.1130)\end{array}$ & $\begin{array}{c}-0.0892^{* * *} \\
(0.0164)\end{array}$ & $\begin{array}{c}-0.1414^{* * *} \\
(0.0469)\end{array}$ & $\begin{array}{c}-0.1363^{* * *} \\
(0.0389)\end{array}$ \\
\hline$\mu_{\text {post }}$ & $\begin{array}{c}0.1131 \\
(0.1042)\end{array}$ & $\begin{array}{c}0.0857 \\
(0.0908)\end{array}$ & $\begin{array}{c}0.1089 \\
(0.1583)\end{array}$ & $\begin{array}{c}0.0169 \\
(0.0215)\end{array}$ & $\begin{array}{c}0.1348 \\
(0.1561)\end{array}$ & $\begin{array}{c}0.1493 \\
(0.1572)\end{array}$ \\
\hline TGARCH $(\gamma)$ & & $\begin{array}{l}0.3171 \text { * } \\
(0.1808)\end{array}$ & & & & \\
\hline EGARCH $(\delta)$ & & & $\begin{array}{l}-0.0788 \\
(0.1228)\end{array}$ & & & \\
\hline $\operatorname{APARCH}(\gamma)$ & & & & $\begin{array}{l}-0.1077 \\
(0.1418)\end{array}$ & & \\
\hline$(\mathrm{A}) \mathrm{CGARCH}(\rho)$ & & & & & $\begin{array}{c}0.8328^{* * *} \\
(0.1183)\end{array}$ & $\begin{array}{c}0.8829 * * * \\
(0.0847) \\
\end{array}$ \\
\hline \multicolumn{7}{|l|}{ Variance Equation } \\
\hline (A)CGARCH $(\theta)$ & & & & & $\begin{array}{c}0.1400 \\
(0.1092)\end{array}$ & $\begin{array}{c}0.1309 \\
(0.0896)\end{array}$ \\
\hline $\operatorname{ACGARCH}(\gamma)$ & & & & & & $\begin{array}{l}-0.0529 \\
(0.1951) \\
\end{array}$ \\
\hline AIC & 2.6339 & 2.6134 & 2.5097 & 2.6367 & 2.5085 & 2.5221 \\
\hline SIC & 2.7522 & 2.7436 & 2.6517 & 2.7668 & 2.6622 & 2.6878 \\
\hline LL & -408.79 & -404.54 & -387.05 & -408.23 & -385.84 & -387.02 \\
\hline JB stat & $\begin{array}{c}137.23 \\
{[0.0000]}\end{array}$ & $\begin{array}{c}133.54 \\
{[0.0000]}\end{array}$ & $\begin{array}{c}94.10 \\
{[0.0000]}\end{array}$ & $\begin{array}{c}80.45 \\
{[0.0000]}\end{array}$ & $\begin{array}{c}138.93 \\
{[0.0000]}\end{array}$ & $\begin{array}{c}138.92 \\
{[0.0000]}\end{array}$ \\
\hline $\mathrm{ARCH}(5)$ & $\begin{array}{c}0.1644 \\
{[0.9754]}\end{array}$ & $\begin{array}{c}0.1136 \\
{[0.9893]}\end{array}$ & $\begin{array}{c}0.0713 \\
{[0.9964]}\end{array}$ & $\begin{array}{c}0.1375 \\
{[0.9835]}\end{array}$ & $\begin{array}{c}0.1831 \\
{[0.9689]}\end{array}$ & $\begin{array}{c}0.1669 \\
{[0.9746]}\end{array}$ \\
\hline$Q^{2}(10)$ & $\begin{array}{c}0.3046 \\
{[0.5810]}\end{array}$ & $\begin{array}{c}0.1472 \\
{[0.7010]}\end{array}$ & $\begin{array}{c}0.0689 \\
{[0.7930]}\end{array}$ & $\begin{array}{c}0.0689 \\
{[0.7930]}\end{array}$ & $\begin{array}{c}0.4737 \\
{[0.4910]}\end{array}$ & $\begin{array}{c}0.3910 \\
{[0.5320]}\end{array}$ \\
\hline
\end{tabular}

Notes: $\left({ }^{*}\right),(* *)$ and $\left(^{* * *}\right)$ denote significance at $10 \%, 5 \%$ and $1 \%$, successively. During the pre-futures period, NYSE is used as the exogeneous variable whereas it is search intensity for the post-futures period, according to PC-LASSO findings. 
Table A8. GARCH-M Tests for Litecoin for the sample of 360 days.

\begin{tabular}{|c|c|c|c|c|c|c|}
\hline & GARCH & TGARCH & EGARCH & APARCH & CGARCH & ACGARCH \\
\hline \multicolumn{7}{|l|}{ Mean Equation } \\
\hline$\lambda_{0}$ & $\begin{array}{c}-0.2591 \text { ** } \\
(0.1023)\end{array}$ & $\begin{array}{c}-0.3346^{* *} \\
(0.1305)\end{array}$ & $\begin{array}{c}-0.2956^{*} \\
(0.1647)\end{array}$ & $\begin{array}{c}-0.0290 \\
(0.1509)\end{array}$ & $\begin{array}{c}0.0143 \\
(0.1212)\end{array}$ & $\begin{array}{l}-0.0695 \\
(0.0986)\end{array}$ \\
\hline$\lambda_{1}$ & $\begin{array}{l}-0.0041 \\
(0.0637)\end{array}$ & $\begin{array}{l}-0.0641 \\
(0.0622)\end{array}$ & $\begin{array}{c}-0.1441^{* * *} \\
(0.0275)\end{array}$ & $\begin{array}{c}-0.0292 \\
(0.0334)\end{array}$ & $\begin{array}{c}-0.1207^{* * *} \\
(0.0399)\end{array}$ & $\begin{array}{c}-0.1217^{* * * *} \\
(0.0433)\end{array}$ \\
\hline$\lambda_{2}$ & $\begin{array}{l}0.2570 * \\
(0.1438)\end{array}$ & $\begin{array}{c}0.3780^{* * *} \\
(0.1828)\end{array}$ & $\begin{array}{c}0.1754 \\
(0.1950)\end{array}$ & $\begin{array}{c}0.0390 \\
(0.1869)\end{array}$ & $\begin{array}{l}-0.1744 \\
(0.1460)\end{array}$ & $\begin{array}{l}-0.0747 \\
(0.1267)\end{array}$ \\
\hline$\kappa_{\text {pre }}$ & $\begin{array}{c}0.0253 \\
(0.0801)\end{array}$ & $\begin{array}{l}-0.0189 \\
(0.0874)\end{array}$ & $\begin{array}{c}-0.1171 \text { ** } \\
(0.0522)\end{array}$ & $\begin{array}{c}-0.1266^{* *} \\
(0.0573)\end{array}$ & $\begin{array}{l}-0.0650 \\
(0.0450)\end{array}$ & $\begin{array}{l}-0.0460 \\
(0.0508)\end{array}$ \\
\hline$\kappa_{\text {post }}$ & $\begin{array}{c}-0.2611 * * * \\
(0.0809)\end{array}$ & $\begin{array}{c}-0.2991 * * * \\
(0.0774)\end{array}$ & $\begin{array}{c}-0.3790^{* * *} \\
(0.0459)\end{array}$ & $\begin{array}{c}-0.2443 * * * \\
(0.0825)\end{array}$ & $\begin{array}{c}-0.3271^{* * *} \\
(0.0494)\end{array}$ & $\begin{array}{c}-0.3104^{* * *} \\
(0.0545)\end{array}$ \\
\hline \multicolumn{7}{|l|}{ Variance Equation } \\
\hline$\omega$ & $\begin{array}{c}0.0965^{* * *} \\
(0.0323)\end{array}$ & $\begin{array}{c}0.1537^{* * *} \\
(0.0344)\end{array}$ & $\begin{array}{c}-0.6286^{* * *} \\
(0.1741)\end{array}$ & $\begin{array}{c}0.0822 * * * \\
(0.0269)\end{array}$ & $\begin{array}{c}0.6497^{* * *} \\
(0.1592)\end{array}$ & $\begin{array}{c}0.5583^{* * *} \\
(0.1470)\end{array}$ \\
\hline $\mathrm{ARCH}(\alpha)$ & $\begin{array}{c}0.2172^{* * *} \\
(0.0530)\end{array}$ & $\begin{array}{c}0.3231^{* * *} \\
(0.0713)\end{array}$ & $\begin{array}{c}0.3897^{* * *} \\
(0.1734)\end{array}$ & $\begin{array}{c}0.1139 * * * \\
(0.0280)\end{array}$ & $\begin{array}{c}0.0748 \\
(0.0718)\end{array}$ & $\begin{array}{l}0.0768 \text { * } \\
(0.0453)\end{array}$ \\
\hline GARCH $(\beta)$ & $\begin{array}{c}0.6795^{* * *} \\
(0.0667)\end{array}$ & $\begin{array}{c}0.6257^{* * *} \\
(0.0665)\end{array}$ & $\begin{array}{c}-0.3831^{* * *} \\
(0.1172)\end{array}$ & $\begin{array}{c}0.8302 * * * \\
(0.0458)\end{array}$ & $\begin{array}{l}-0.3088 \\
(0.7181)\end{array}$ & $\begin{array}{c}0.6554 * * * \\
(0.1820)\end{array}$ \\
\hline$\mu_{\text {pre }}$ & $\begin{array}{c}0.1961^{* * *} \\
(0.0395)\end{array}$ & $\begin{array}{c}0.2489 * * * \\
(0.0299)\end{array}$ & $\begin{array}{c}0.2828 * * \\
(0.1221)\end{array}$ & $\begin{array}{c}0.0649^{* * *} \\
(0.0100)\end{array}$ & $\begin{array}{l}0.1090^{* *} \\
(0.0503)\end{array}$ & $\begin{array}{c}0.1565^{* * *} \\
(0.0283)\end{array}$ \\
\hline$\mu_{\text {post }}$ & $\begin{array}{c}0.0853 \\
(0.0888)\end{array}$ & $\begin{array}{c}0.0959 \\
(0.0979)\end{array}$ & $\begin{array}{c}0.1969 \\
(0.1561)\end{array}$ & $\begin{array}{c}0.0597^{* *} \\
(0.0261)\end{array}$ & $\begin{array}{c}0.2085^{* * *} \\
(0.0751)\end{array}$ & $\begin{array}{c}0.2068^{* *} \\
(0.0978)\end{array}$ \\
\hline TGARCH $(\gamma)$ & & $\begin{array}{c}-0.2961^{* * *} \\
(0.0833\end{array}$ & & & & \\
\hline EGARCH $(\delta)$ & & & $\begin{array}{c}0.1859 \\
(0.1357)\end{array}$ & & & \\
\hline $\operatorname{APARCH}(\gamma)$ & & & & $\begin{array}{c}-0.6354 * * * \\
(0.1680)\end{array}$ & & \\
\hline (A)CGARCH $(\rho)$ & & & & & $\begin{array}{c}0.9553 * * * \\
(0.0248)\end{array}$ & $\begin{array}{c}0.9822^{* * *} \\
(0.0010)\end{array}$ \\
\hline (A)CGARCH $(\theta)$ & & & & & $\begin{array}{l}0.0355^{*} \\
(0.0207)\end{array}$ & $\begin{array}{l}-0.0063 \\
(0.0089)\end{array}$ \\
\hline $\operatorname{ACGARCH}(\gamma)$ & & & & & & $\begin{array}{c}0.1005 \\
(0.1129)\end{array}$ \\
\hline AIC & 2.5709 & 2.5505 & 2.4590 & 2.5828 & 2.4463 & 2.4261 \\
\hline SIC & 2.6791 & 2.6694 & 2.5888 & 2.7018 & 2.5869 & 2.5775 \\
\hline LL & -451.48 & -446.81 & -429.39 & -452.61 & -426.11 & -421.48 \\
\hline JB stat & $\begin{array}{l}163.564 \\
{[0.0000]}\end{array}$ & $\begin{array}{l}144.089 \\
{[0.0000]}\end{array}$ & $\begin{array}{l}1134.740 \\
{[0.0000]}\end{array}$ & $\begin{array}{l}162.583 \\
{[0.0000]}\end{array}$ & $\begin{array}{l}372.786 \\
{[0.0000]}\end{array}$ & $\begin{array}{l}171.846 \\
{[0.0000]}\end{array}$ \\
\hline $\mathrm{ARCH}(5)$ & $\begin{array}{c}0.6315 \\
{[0.6758]}\end{array}$ & $\begin{array}{c}0.9938 \\
{[0.4214]}\end{array}$ & $\begin{array}{c}4.6074 \\
{[0.0004]}\end{array}$ & $\begin{array}{c}1.1945 \\
{[0.3114]}\end{array}$ & $\begin{array}{c}0.9658 \\
{[0.4387]}\end{array}$ & $\begin{array}{c}0.8211 \\
{[0.5353]}\end{array}$ \\
\hline$Q^{2}(10)$ & $\begin{array}{c}0.1111 \\
{[0.7390]}\end{array}$ & $\begin{array}{c}0.3153 \\
{[0.5740]}\end{array}$ & $\begin{array}{c}0.1323 \\
{[0.7160]}\end{array}$ & $\begin{array}{c}0.1113 \\
{[0.7390]}\end{array}$ & $\begin{array}{c}0.7483 \\
{[0.3870]}\end{array}$ & $\begin{array}{c}0.1809 \\
{[0.6710]}\end{array}$ \\
\hline
\end{tabular}

Notes: $\left({ }^{*}\right),(* *)$ and $(* * *)$ denote significance at $10 \%, 5 \%$ and $1 \%$, successively. Both for the pre and post-futures period, search intensity is used as the exogenous variable, according to PC-LASSO findings.

\section{Appendix B. Recovery Period Analyses}

Table A9. PC-LASSO coefficients for recovery period.

\begin{tabular}{lccccc}
\hline Variable & Bitcoin & Ethereum & Ripple & Bitcoin Cash & Litecoin \\
\hline$€ / \$$ & 0.00000 & 0.00000 & 0.00000 & 0.00000 & 0.00000 \\
Gold & 0.00000 & 0.00000 & 0.00000 & 0.00000 & 0.00000 \\
NYSE & 0.00000 & 0.00000 & 0.00000 & -0.01234 & 0.00000 \\
Oil price & 0.00000 & 0.00000 & 0.00000 & -0.01177 & 0.00000 \\
GTrends & $\mathbf{0 . 1 2 7 6 7}$ & $\mathbf{0 . 0 7 8 4 0}$ & $\mathbf{0 . 0 8 4 6 3}$ & $\mathbf{0 . 1 0 8 8 7}$ & $\mathbf{0 . 0 6 4 1 2}$ \\
China & 0.00000 & 0.03764 & 0.00000 & 0.00000 & 0.00097 \\
VIX & 0.00000 & 0.00000 & 0.00000 & 0.00000 & 0.00000 \\
FFR & 0.00000 & 0.00000 & 0.00000 & 0.00000 & 0.00000 \\
EPU & 0.00000 & 0.00000 & 0.00168 & 0.00000 & 0.03764 \\
\hline
\end{tabular}

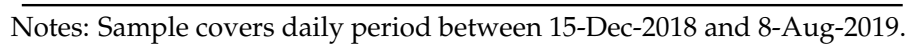


Table A10. GARCH-M Tests for Bitcoin for the recovery period.

\begin{tabular}{|c|c|c|c|c|c|c|}
\hline & GARCH & TGARCH & EGARCH & APARCH & CGARCH & ACGARCH \\
\hline \multicolumn{7}{|l|}{ Mean Equation } \\
\hline$\lambda_{0}$ & $\begin{array}{c}0.4170^{* * *} \\
(0.1128)\end{array}$ & $\begin{array}{l}0.2853 * \\
(0.1701)\end{array}$ & $\begin{array}{c}-0.2034^{* * *} \\
(0.0447)\end{array}$ & $\begin{array}{c}-0.1562 * * * \\
(0.0464)\end{array}$ & $\begin{array}{c}-0.2102 * \\
(0.1187)\end{array}$ & $\begin{array}{c}-0.2223 * \\
(0.1182)\end{array}$ \\
\hline$\lambda_{1}$ & $\begin{array}{l}-0.0402 \\
(0.0487)\end{array}$ & $\begin{array}{c}-0.0915^{* *} \\
(0.0455)\end{array}$ & $\begin{array}{c}-0.1196^{* *} \\
(0.0496)\end{array}$ & $\begin{array}{c}-0.1488^{* *} \\
(0.0738)\end{array}$ & $\begin{array}{c}-0.1289 * * \\
(0.0542)\end{array}$ & $\begin{array}{c}-0.1189 * * \\
(0.0469)\end{array}$ \\
\hline$\lambda_{2}$ & $\begin{array}{c}-0.5829 * * * \\
(0.1413)\end{array}$ & $\begin{array}{c}-0.4114 \\
(0.2227)\end{array}$ & $\begin{array}{c}0.2869^{* * *} \\
(0.0972)\end{array}$ & $\begin{array}{c}0.1657 \\
(0.1109)\end{array}$ & $\begin{array}{l}0.2905^{*} \\
(0.1610)\end{array}$ & $\begin{array}{l}0.2947^{*} \\
(0.1582)\end{array}$ \\
\hline$\kappa$ & $\begin{array}{c}0.3929 * * * \\
(0.0703)\end{array}$ & $\begin{array}{c}0.3087^{* * * *} \\
(0.0753)\end{array}$ & $\begin{array}{c}0.1316^{* * * *} \\
(0.0386)\end{array}$ & $\begin{array}{l}0.0774 \text { * } \\
(0.0466)\end{array}$ & $\begin{array}{c}0.2140^{* * *} \\
(0.0493)\end{array}$ & $\begin{array}{c}0.1964^{* * * *} \\
(0.0493)\end{array}$ \\
\hline \multicolumn{7}{|l|}{ Variance Equation } \\
\hline$\omega$ & $\begin{array}{c}0.2883^{* * *} \\
(0.0355)\end{array}$ & $\begin{array}{c}0.2987^{* * *} \\
(0.0380)\end{array}$ & $\begin{array}{c}0.0164^{* * *} \\
(0.0061)\end{array}$ & $\begin{array}{c}0.0061 \\
(0.0044)\end{array}$ & $\begin{array}{c}0.6080^{* * *} \\
(0.2184)\end{array}$ & $\begin{array}{c}0.5983^{* * *} \\
(0.1820)\end{array}$ \\
\hline $\mathrm{ARCH}(\alpha)$ & $\begin{array}{c}0.1649 * * * \\
(0.0317)\end{array}$ & $\begin{array}{c}0.1241^{* * * *} \\
(0.0340)\end{array}$ & $\begin{array}{c}-0.0385^{* * *} \\
(0.0098)\end{array}$ & $\begin{array}{c}0.0307^{* * *} \\
(0.0092)\end{array}$ & $\begin{array}{l}-0.0265 \\
(0.0927)\end{array}$ & $\begin{array}{l}-0.0940 \\
(0.0915)\end{array}$ \\
\hline GARCH $(\beta)$ & $\begin{array}{c}0.4425^{* * *} \\
(0.0500)\end{array}$ & $\begin{array}{c}0.4513^{* * *} \\
(0.0467)\end{array}$ & $\begin{array}{c}0.9953^{* * *} \\
(0.0057)\end{array}$ & $\begin{array}{c}0.9723^{* * *} \\
(0.0097)\end{array}$ & $\begin{array}{l}-0.3160 \\
(2.6136)\end{array}$ & $\begin{array}{c}0.8987^{* * *} \\
(0.3353)\end{array}$ \\
\hline$\mu$ & $\begin{array}{c}0.4401^{* * *} \\
(0.0378)\end{array}$ & $\begin{array}{c}0.4229 * * * \\
(0.0314)\end{array}$ & $\begin{array}{c}0.5865^{* * *} \\
(0.0909)\end{array}$ & $\begin{array}{c}0.1269^{* * *} \\
(0.0144)\end{array}$ & $\begin{array}{c}0.2188^{* * *} \\
(0.0618)\end{array}$ & $\begin{array}{c}0.2512 * * * \\
(0.0658)\end{array}$ \\
\hline TGARCH $(\gamma)$ & & $\begin{array}{l}-0.0374 \\
(0.0534)\end{array}$ & & & & \\
\hline EGARCH $(\delta)$ & & & $\begin{array}{l}-0.0824 \\
(0.0523)\end{array}$ & & & \\
\hline $\operatorname{APARCH}(\gamma)$ & & & & $\begin{array}{c}0.9463^{* * *} \\
(0.2114)\end{array}$ & & \\
\hline \multicolumn{7}{|l|}{ Variance Equation } \\
\hline (A)CGARCH $(\rho)$ & & & & & $\begin{array}{c}0.9338 * * * \\
(0.0286)\end{array}$ & $\begin{array}{c}0.9356^{* * *} \\
(0.0260)\end{array}$ \\
\hline (A)CGARCH $(\theta)$ & & & & & $\begin{array}{c}0.0751 \\
(0.0478)\end{array}$ & $\begin{array}{c}0.0699 \\
(0.0936)\end{array}$ \\
\hline $\operatorname{ACGARCH}(\gamma)$ & & & & & & $\begin{array}{c}0.0303 \\
(0.0929)\end{array}$ \\
\hline $\mathrm{AIC}$ & 2.4232 & 2.4650 & 2.0630 & 2.1581 & 2.2378 & 2.2266 \\
\hline SIC & 2.5406 & 2.5971 & 2.2098 & 2.2902 & 2.3993 & 2.4027 \\
\hline LL & -277.93 & -281.86 & -233.44 & -245.65 & -253.06 & -250.73 \\
\hline JB stat & 16.16 & 35.23 & 297.92 & 11.07 & 22.71 & 24.12 \\
\hline & {$[0.0003]$} & {$[0.0000]$} & {$[0.0000]$} & {$[0.0039]$} & {$[0.0000]$} & [0.0000] \\
\hline $\mathrm{ARCH}(5)$ & 2.6733 & 6.4518 & 0.4024 & 1.0228 & 0.1956 & 1.4488 \\
\hline & {$[0.0227]$} & {$[0.0000]$} & [0.8469] & {$[0.4050]$} & {$[0.9640]$} & [0.2078] \\
\hline$Q^{2}(10)$ & $\begin{array}{c}0.7854 \\
{[0.3760]}\end{array}$ & $\begin{array}{c}0.3883 \\
{[0.5330]}\end{array}$ & $\begin{array}{c}1.5919 \\
{[0.2070]}\end{array}$ & $\begin{array}{c}0.4933 \\
{[0.4820]}\end{array}$ & $\begin{array}{c}0.1204 \\
{[0.7290]}\end{array}$ & $\begin{array}{c}0.1141 \\
{[0.7350]}\end{array}$ \\
\hline
\end{tabular}

Notes: $\left({ }^{*}\right),(* *)$ and $\left({ }^{* *}\right)$ denote significance at $10 \%, 5 \%$ and $1 \%$, successively. Same notes as in Table A9. 
Table A11. GARCH-M Tests for Ethereum for the recovery period.

\begin{tabular}{|c|c|c|c|c|c|c|}
\hline & GARCH & TGARCH & EGARCH & APARCH & CGARCH & ACGARCH \\
\hline \multicolumn{7}{|l|}{ Mean Equation } \\
\hline$\lambda_{0}$ & $\begin{array}{c}-0.2035^{* * *} \\
(0.0785)\end{array}$ & $\begin{array}{c}-0.2195^{* * *} \\
(0.0427)\end{array}$ & $\begin{array}{c}-0.1775^{* *} \\
(0.0814)\end{array}$ & $\begin{array}{l}-0.1501 \\
(0.0895)\end{array}$ & $\begin{array}{c}-0.2376^{* *} \\
(0.1130)\end{array}$ & $\begin{array}{c}-0.2310 * * \\
(0.1067)\end{array}$ \\
\hline$\lambda_{1}$ & $\begin{array}{c}-0.1447 * \\
(0.0791)\end{array}$ & $\begin{array}{c}-0.1403^{*} \\
(0.0730)\end{array}$ & $\begin{array}{c}-0.1867^{* * *} \\
(0.0462)\end{array}$ & $\begin{array}{c}-0.1609 * * \\
(0.0662)\end{array}$ & $\begin{array}{c}-0.1786^{* * *} \\
(0.0499)\end{array}$ & $\begin{array}{c}-0.1743^{* * *} \\
(0.0459)\end{array}$ \\
\hline$\lambda_{2}$ & $\begin{array}{l}0.2071 * \\
(0.1105)\end{array}$ & $\begin{array}{c}0.2351 * * \\
(0.0984)\end{array}$ & $\begin{array}{c}0.1266 \\
(0.1187)\end{array}$ & $\begin{array}{c}0.1640 \\
(0.1365)\end{array}$ & $\begin{array}{c}0.2266 \\
(0.1448)\end{array}$ & $\begin{array}{c}0.1975 \\
(0.1352)\end{array}$ \\
\hline$\kappa$ & $\begin{array}{l}0.1189 * \\
(0.0678)\end{array}$ & $\begin{array}{l}0.1065^{*} \\
(0.0636)\end{array}$ & $\begin{array}{c}0.0666 \\
(0.0436)\end{array}$ & $\begin{array}{l}0.1046^{*} \\
(0.0568)\end{array}$ & $\begin{array}{c}0.1393^{* * *} \\
(0.0466)\end{array}$ & $\begin{array}{c}0.1103 * * \\
(0.0469)\end{array}$ \\
\hline \multicolumn{7}{|l|}{ Variance Equation } \\
\hline$\omega$ & $\begin{array}{c}0.0379 * * \\
(0.0179)\end{array}$ & $\begin{array}{l}0.0266^{*} \\
(0.0152)\end{array}$ & $\begin{array}{l}0.0219 * \\
(0.0127)\end{array}$ & $\begin{array}{c}-0.0117^{*} \\
(0.0060)\end{array}$ & $\begin{array}{c}0.5357^{* * * *} \\
(0.0836)\end{array}$ & $\begin{array}{c}0.5919 * * * \\
(0.2265)\end{array}$ \\
\hline $\mathrm{ARCH}(\alpha)$ & $\begin{array}{c}0.0907^{* * *} \\
(0.0296)\end{array}$ & $\begin{array}{l}0.0569 * * \\
(0.0232)\end{array}$ & $\begin{array}{c}-0.0387^{* * *} \\
(0.0141)\end{array}$ & $\begin{array}{c}0.0153 \\
(0.0259)\end{array}$ & $\begin{array}{c}0.0805 \\
(0.1146)\end{array}$ & $\begin{array}{c}0.0610 \\
(0.0938)\end{array}$ \\
\hline $\operatorname{GARCH}(\beta)$ & $\begin{array}{c}0.8586^{* * *} \\
(0.0371)\end{array}$ & $\begin{array}{c}0.8947^{* * *} \\
(0.0334)\end{array}$ & $\begin{array}{c}1.0114^{* * *} \\
(0.0064)\end{array}$ & $\begin{array}{c}1.0003^{* * * *} \\
(0.0185)\end{array}$ & $\begin{array}{c}0.2420 \\
(0.6634)\end{array}$ & $\begin{array}{c}0.8529 * * * \\
(0.2367)\end{array}$ \\
\hline$\mu$ & $\begin{array}{c}0.2575^{* * *} \\
(0.0365)\end{array}$ & $\begin{array}{c}0.2397^{* * * *} \\
(0.0391)\end{array}$ & $\begin{array}{c}0.3568^{* * *} \\
(0.0660)\end{array}$ & $\begin{array}{c}0.0824^{* * *} \\
(0.0080)\end{array}$ & $\begin{array}{c}0.1773^{* * *} \\
(0.0558)\end{array}$ & $\begin{array}{c}0.2032^{* * *} \\
(0.0787)\end{array}$ \\
\hline TGARCH $(\gamma)$ & & $\begin{array}{c}0.0109 \\
(0.0223)\end{array}$ & & & & \\
\hline EGARCH $(\delta)$ & & & $\begin{array}{c}0.0264 \\
(0.0425)\end{array}$ & & & \\
\hline $\operatorname{APARCH}(\gamma)$ & & & & $\begin{array}{l}0.8462 * \\
(0.4909)\end{array}$ & & \\
\hline (A)CGARCH $(\rho)$ & & & & & $\begin{array}{c}0.9785^{* * *} \\
(0.0018)\end{array}$ & $\begin{array}{c}0.9730^{* * *} \\
(0.0144)\end{array}$ \\
\hline (A)CGARCH $(\theta)$ & & & & & $\begin{array}{l}-0.0123 \\
(0.0219)\end{array}$ & $\begin{array}{l}-0.0055 \\
(0.0553)\end{array}$ \\
\hline $\operatorname{ACGARCH}(\gamma)$ & & & & & & $\begin{array}{l}-0.0392 \\
(0.0842)\end{array}$ \\
\hline AIC & 2.6108 & 2.6056 & 2.3896 & 2.4999 & 2.4252 & 2.4305 \\
\hline SIC & 2.7282 & 2.7377 & 2.5364 & 2.6320 & 2.5867 & 2.6066 \\
\hline LL & -300.07 & -298.46 & -271.98 & -285.98 & -275.18 & -274.79 \\
\hline JB stat & 54.14 & 52.86 & 191.67 & 13.41 & 34.27 & 40.47 \\
\hline & {$[0.0000]$} & {$[0.0000]$} & {$[0.0000]$} & {$[0.0012]$} & {$[0.0000]$} & {$[0.0000]$} \\
\hline $\mathrm{ARCH}(5)$ & 0.2800 & 0.1706 & 2.1382 & 0.3198 & 0.6598 & 0.3411 \\
\hline & {$[0.9238]$} & [0.9733] & [0.0619] & [0.9008] & [0.6543] & [0.8876] \\
\hline$Q^{2}(10)$ & 0.2520 & 0.0423 & 8.5346 & 0.1539 & 0.0277 & 0.0224 \\
\hline & {$[0.6160]$} & {$[0.8370]$} & {$[0.0030]$} & {$[0.6950]$} & {$[0.8680]$} & {$[0.8810]$} \\
\hline
\end{tabular}


Table A12. GARCH-M Tests for Ripple for the recovery period.

\begin{tabular}{|c|c|c|c|c|c|c|}
\hline & GARCH & TGARCH & EGARCH & APARCH & CGARCH & ACGARCH \\
\hline \multicolumn{7}{|l|}{ Mean Equation } \\
\hline$\lambda_{0}$ & $\begin{array}{c}-0.1765^{* * *} \\
(0.0671)\end{array}$ & $\begin{array}{c}-0.2225^{* * *} \\
(0.0342)\end{array}$ & $\begin{array}{c}-0.1429 * * * \\
(0.0554)\end{array}$ & $\begin{array}{c}-0.1293^{* * *} \\
(0.0463)\end{array}$ & $\begin{array}{c}-0.2057^{*} \\
(0.1109)\end{array}$ & $\begin{array}{c}-0.2234^{* *} \\
(0.1027)\end{array}$ \\
\hline$\lambda_{1}$ & $\begin{array}{c}-0.1838^{* * *} \\
(0.0692)\end{array}$ & $\begin{array}{c}-0.1641 \text { ** } \\
(0.0776)\end{array}$ & $\begin{array}{c}-0.2038^{* * *} \\
(0.0514)\end{array}$ & $\begin{array}{c}-0.2528 \text { *** } \\
(0.0442)\end{array}$ & $\begin{array}{c}-0.2280 * * * \\
(0.0623)\end{array}$ & $\begin{array}{c}-0.2265^{* * *} \\
(0.0585)\end{array}$ \\
\hline$\lambda_{2}$ & $\begin{array}{l}0.2086^{*} \\
(0.1123)\end{array}$ & $\begin{array}{c}0.2670 * * * \\
(0.0797)\end{array}$ & $\begin{array}{c}0.1651^{* *} \\
(0.0841)\end{array}$ & $\begin{array}{c}0.0602 \\
(0.0438)\end{array}$ & $\begin{array}{c}0.2838 \\
(0.1767)\end{array}$ & $\begin{array}{l}0.3197^{*} \\
(0.1701)\end{array}$ \\
\hline$\kappa$ & $\begin{array}{c}0.1014 \\
(0.0695)\end{array}$ & $\begin{array}{c}0.0832 \\
(0.0594)\end{array}$ & $\begin{array}{c}0.0664 \\
(0.0434)\end{array}$ & $\begin{array}{c}0.0131 \\
(0.0533)\end{array}$ & $\begin{array}{c}0.1272 * * * \\
(0.0468)\end{array}$ & $\begin{array}{c}0.1071^{* *} \\
(0.0469)\end{array}$ \\
\hline \multicolumn{7}{|l|}{ Variance Equation } \\
\hline$\omega$ & $\begin{array}{c}0.0673^{* * *} \\
(0.0194)\end{array}$ & $\begin{array}{c}0.1285^{* * *} \\
(0.0296)\end{array}$ & $\begin{array}{c}0.0532 * * * \\
(0.0008)\end{array}$ & $\begin{array}{l}0.0256^{* *} \\
(0.0115)\end{array}$ & $\begin{array}{c}0.3265^{* * *} \\
(0.0714)\end{array}$ & $\begin{array}{c}0.2899 * * * \\
(0.0056)\end{array}$ \\
\hline $\mathrm{ARCH}(\alpha)$ & $\begin{array}{c}0.0813^{* *} \\
(0.0362)\end{array}$ & $\begin{array}{c}0.0992 \\
(0.0666)\end{array}$ & $\begin{array}{c}-0.0952^{* * *} \\
(0.0046)\end{array}$ & $\begin{array}{c}0.1123^{* * *} \\
(0.0291)\end{array}$ & $\begin{array}{l}0.1259 * \\
(0.0698)\end{array}$ & $\begin{array}{c}0.0367 \\
(0.0665)\end{array}$ \\
\hline $\operatorname{GARCH}(\beta)$ & $\begin{array}{c}0.8110^{* * *} \\
(0.0467)\end{array}$ & $\begin{array}{c}0.6376^{* * * *} \\
(0.0596)\end{array}$ & $\begin{array}{c}0.9899 * * * \\
(0.0073)\end{array}$ & $\begin{array}{c}0.8918^{* * * *} \\
(0.0260)\end{array}$ & $\begin{array}{c}0.4824 \\
(0.3141)\end{array}$ & $\begin{array}{c}0.6058 \\
(0.3766)\end{array}$ \\
\hline$\mu$ & $\begin{array}{c}0.2843^{* * *} \\
(0.0317)\end{array}$ & $\begin{array}{c}0.3420 * * * \\
(0.0374)\end{array}$ & $\begin{array}{c}0.5179 * * * \\
(0.0458)\end{array}$ & $\begin{array}{c}0.1591^{* * *} \\
(0.0164)\end{array}$ & $\begin{array}{c}0.1469 * * * \\
(0.0327)\end{array}$ & $\begin{array}{c}0.1366^{* * *} \\
(0.0313)\end{array}$ \\
\hline TGARCH $(\gamma)$ & & $\begin{array}{l}0.1726^{*} \\
(0.1000)\end{array}$ & & & & \\
\hline EGARCH $(\delta)$ & & & $\begin{array}{c}-0.1243^{* * *} \\
(0.0447)\end{array}$ & & & \\
\hline $\operatorname{APARCH}(\gamma)$ & & & & $\begin{array}{c}0.7351 * * * \\
(0.1863)\end{array}$ & & \\
\hline (A)CGARCH $(\rho)$ & & & & & $\begin{array}{c}0.9378 * * * \\
(0.0037)\end{array}$ & $\begin{array}{c}0.9303 * * * \\
(0.0237)\end{array}$ \\
\hline (A)CGARCH $(\theta)$ & & & & & $\begin{array}{c}0.0327 \\
(0.0287)\end{array}$ & $\begin{array}{c}0.0363 \\
(0.0354)\end{array}$ \\
\hline $\operatorname{ACGARCH}(\gamma)$ & & & & & & $\begin{array}{c}0.0551 \\
(0.0869)\end{array}$ \\
\hline AIC & 2.4175 & 2.4266 & 2.1666 & 2.2814 & 2.3181 & 2.2987 \\
\hline SIC & 2.5349 & 2.5587 & 2.3134 & 2.4135 & 2.4795 & 2.4748 \\
\hline LL & -277.27 & -277.34 & -245.66 & -260.21 & -262.53 & -259.24 \\
\hline JB stat & $\begin{array}{c}79.59 \\
{[0.0000]}\end{array}$ & $\begin{array}{c}70.53 \\
{[0.0000]}\end{array}$ & $\begin{array}{c}362.77 \\
{[0.0000]}\end{array}$ & $\begin{array}{c}6.93 \\
{[0.0313]}\end{array}$ & $\begin{array}{c}147.43 \\
{[0.0000]}\end{array}$ & $\begin{array}{c}173.41 \\
{[0.0000]}\end{array}$ \\
\hline $\mathrm{ARCH}(5)$ & $\begin{array}{c}0.6538 \\
{[0.6589]}\end{array}$ & $\begin{array}{c}0.7382 \\
{[0.5956]}\end{array}$ & $\begin{array}{c}3.5939 \\
{[0.0038]}\end{array}$ & $\begin{array}{c}0.8075 \\
{[0.5453]}\end{array}$ & $\begin{array}{c}0.3923 \\
{[0.8538]}\end{array}$ & $\begin{array}{c}0.4899 \\
{[0.7837]}\end{array}$ \\
\hline$Q^{2}(10)$ & $\begin{array}{c}0.5331 \\
{[0.4650]}\end{array}$ & $\begin{array}{c}0.0374 \\
{[0.8470]}\end{array}$ & $\begin{array}{l}11.4740 \\
{[0.0010]}\end{array}$ & $\begin{array}{c}0.5719 \\
{[0.4490]}\end{array}$ & $\begin{array}{c}0.0107 \\
{[0.9170]}\end{array}$ & $\begin{array}{c}0.3417 \\
{[0.5590]}\end{array}$ \\
\hline
\end{tabular}

Notes: $\left({ }^{*}\right),(* *)$ and $\left({ }^{* *}\right)$ denote significance at $10 \%, 5 \%$ and $1 \%$, successively. Same notes as in Table A9. 
Table A13. GARCH-M Tests for Bitcoin Cash for the recovery period.

\begin{tabular}{|c|c|c|c|c|c|c|}
\hline & GARCH & TGARCH & EGARCH & APARCH & CGARCH & ACGARCH \\
\hline \multicolumn{7}{|l|}{ Mean Equation } \\
\hline$\lambda_{0}$ & $\begin{array}{l}-0.2111 \\
(0.1840)\end{array}$ & $\begin{array}{l}-0.2262 \\
(0.1725)\end{array}$ & $\begin{array}{c}-0.5563^{* * *} \\
(0.1284)\end{array}$ & $\begin{array}{l}-0.1677 \\
(0.1157)\end{array}$ & $\begin{array}{c}-0.4240^{* * *} \\
(0.0862)\end{array}$ & $\begin{array}{c}-0.4109^{* * *} \\
(0.1056)\end{array}$ \\
\hline$\lambda_{1}$ & $\begin{array}{c}-0.1564 \text { * } \\
(0.0900)\end{array}$ & $\begin{array}{l}-0.1327 \\
(0.0973)\end{array}$ & $\begin{array}{c}-0.1912^{* * *} \\
(0.0240)\end{array}$ & $\begin{array}{c}-0.1406^{* * *} \\
(0.0542)\end{array}$ & $\begin{array}{c}-0.1818^{* * *} \\
(0.0306)\end{array}$ & $\begin{array}{c}-0.1446^{* * *} \\
(0.0379)\end{array}$ \\
\hline$\lambda_{2}$ & $\begin{array}{c}0.1940 \\
(0.2768)\end{array}$ & $\begin{array}{c}0.2124 \\
(0.2668)\end{array}$ & $\begin{array}{c}0.6017^{* * * *} \\
(0.1799)\end{array}$ & $\begin{array}{c}0.1159 \\
(0.1966)\end{array}$ & $\begin{array}{c}0.3802^{* * *} \\
(0.1062)\end{array}$ & $\begin{array}{c}0.4311^{* * * *} \\
(0.1458)\end{array}$ \\
\hline$\kappa$ & $\begin{array}{c}0.1499 * * * \\
(0.0494)\end{array}$ & $\begin{array}{c}0.1352 * * * \\
(0.0500)\end{array}$ & $\begin{array}{l}-0.0638 \\
(0.0459)\end{array}$ & $\begin{array}{c}0.0233 \\
(0.0695)\end{array}$ & $\begin{array}{l}-0.0005 \\
(0.0173)\end{array}$ & $\begin{array}{c}0.0386 \\
(0.0388)\end{array}$ \\
\hline \multicolumn{7}{|l|}{ Variance Equation } \\
\hline$\omega$ & $\begin{array}{c}0.2277^{* * *} \\
(0.0436)\end{array}$ & $\begin{array}{c}0.1930 * * * \\
(0.0387)\end{array}$ & $\begin{array}{c}-0.5416^{* * *} \\
(0.1130)\end{array}$ & $\begin{array}{c}0.0575^{* * *} \\
(0.0121)\end{array}$ & $\begin{array}{c}0.6733^{* * *} \\
(0.2453)\end{array}$ & $\begin{array}{c}0.6241 * * * \\
(0.1464)\end{array}$ \\
\hline $\mathrm{ARCH}(\alpha)$ & $\begin{array}{c}0.3782 * * * \\
(0.0804)\end{array}$ & $\begin{array}{c}0.2911 * * * \\
(0.0596)\end{array}$ & $\begin{array}{c}0.4422 * * * \\
(0.1066)\end{array}$ & $\begin{array}{c}0.0406 * * \\
(0.0217)\end{array}$ & $\begin{array}{c}0.2773 * * \\
(0.1270)\end{array}$ & $\begin{array}{c}0.0662 \\
(0.0821)\end{array}$ \\
\hline GARCH $(\beta)$ & $\begin{array}{c}0.3272 * * * \\
(0.0851)\end{array}$ & $\begin{array}{c}0.3941^{* * * *} \\
(0.0873)\end{array}$ & $\begin{array}{c}0.5845^{* * *} \\
(0.0846)\end{array}$ & $\begin{array}{c}0.9059^{* * *} \\
(0.0186)\end{array}$ & $\begin{array}{l}-0.0356 \\
(0.1541)\end{array}$ & $\begin{array}{l}-0.1009 \\
(0.1195)\end{array}$ \\
\hline$\mu$ & $\begin{array}{c}0.2839 * * * \\
(0.0345)\end{array}$ & $\begin{array}{c}0.2778^{* * *} \\
(0.0344)\end{array}$ & $\begin{array}{c}0.6286^{* * *} \\
(0.1053)\end{array}$ & $\begin{array}{c}0.1217^{* * *} \\
(0.0200)\end{array}$ & $\begin{array}{c}0.2037 * * * \\
(0.0771)\end{array}$ & $\begin{array}{c}0.1853 * * * \\
(0.0631)\end{array}$ \\
\hline TGARCH $(\gamma)$ & & $\begin{array}{c}0.1419 \\
(0.1403)\end{array}$ & & & & \\
\hline EGARCH $(\delta)$ & & & $\begin{array}{l}-0.0410 \\
(0.0710)\end{array}$ & & & \\
\hline $\operatorname{APARCH}(\gamma)$ & & & & $\begin{array}{c}0.9941^{* * *} \\
(0.0675)\end{array}$ & & \\
\hline (A)CGARCH $(\rho)$ & & & & & $\begin{array}{c}0.8414^{* * *} \\
(0.0656)\end{array}$ & $\begin{array}{c}0.8337^{* * *} \\
(0.0674)\end{array}$ \\
\hline (A)CGARCH $(\theta)$ & & & & & $\begin{array}{c}0.0569 \\
(0.0937)\end{array}$ & $\begin{array}{c}0.0021 \\
(0.0319)\end{array}$ \\
\hline $\operatorname{ACGARCH}(\gamma)$ & & & & & & $\begin{array}{c}0.3304^{* *} \\
(0.1424)\end{array}$ \\
\hline AIC & 2.3033 & 2.3107 & 2.0289 & 2.1924 & 2.0589 & 2.0692 \\
\hline SIC & 2.4207 & 2.4428 & 2.1757 & 2.3245 & 2.2204 & 2.2453 \\
\hline LL & -263.79 & -263.67 & -229.41 & -249.70 & -231.95 & -232.17 \\
\hline JB stat & $\begin{array}{c}43.14 \\
{[0.0000]}\end{array}$ & $\begin{array}{c}46.60 \\
{[0.0000]}\end{array}$ & $\begin{array}{c}126.81 \\
{[0.0000]}\end{array}$ & $\begin{array}{c}50.92 \\
{[0.0000]}\end{array}$ & $\begin{array}{c}93.88 \\
{[0.0000]}\end{array}$ & $\begin{array}{c}114.54 \\
{[0.0000]}\end{array}$ \\
\hline $\mathrm{ARCH}(5)$ & $\begin{array}{c}1.1687 \\
{[0.3255]}\end{array}$ & $\begin{array}{c}1.2851 \\
{[0.2712]}\end{array}$ & $\begin{array}{c}0.4671 \\
{[0.8005]}\end{array}$ & $\begin{array}{c}0.4418 \\
{[0.8190]}\end{array}$ & $\begin{array}{c}0.7987 \\
{[0.5516]}\end{array}$ & $\begin{array}{c}0.4301 \\
{[0.8274]}\end{array}$ \\
\hline$Q^{2}(10)$ & $\begin{array}{c}0.8169 \\
{[0.3660]}\end{array}$ & $\begin{array}{c}0.4563 \\
{[0.4990]}\end{array}$ & $\begin{array}{c}0.2478 \\
{[0.6190]}\end{array}$ & $\begin{array}{c}0.6182 \\
{[0.4320]}\end{array}$ & $\begin{array}{c}1.0492 \\
{[0.3060]}\end{array}$ & $\begin{array}{c}0.3069 \\
{[0.5800]}\end{array}$ \\
\hline
\end{tabular}

Notes: $\left({ }^{*}\right),\left({ }^{* *}\right)$ and $\left({ }^{* *}\right)$ denote significance at $10 \%, 5 \%$ and $1 \%$, successively. Same notes as in Table A9. 
Table A14. GARCH-M Tests for Litecoin for the recovery period.

\begin{tabular}{|c|c|c|c|c|c|c|}
\hline & GARCH & TGARCH & EGARCH & APARCH & CGARCH & ACGARCH \\
\hline \multicolumn{7}{|l|}{ Mean Equation } \\
\hline$\lambda_{1}$ & $\begin{array}{l}-0.0713 \\
(0.0611)\end{array}$ & $\begin{array}{l}-0.0241 \\
(0.0679)\end{array}$ & $\begin{array}{l}-0.0111 \\
(0.0714)\end{array}$ & $\begin{array}{l}-0.0584 \\
(0.0484)\end{array}$ & $\begin{array}{l}-0.0675 \\
(0.0687)\end{array}$ & $\begin{array}{c}-0.1011^{* * * *} \\
(0.0128)\end{array}$ \\
\hline$\lambda_{2}$ & $\begin{array}{c}-0.0973 * \\
(0.0526)\end{array}$ & $\begin{array}{l}-0.0140 \\
(0.0739)\end{array}$ & $\begin{array}{c}0.5668 \\
(0.4937)\end{array}$ & $\begin{array}{l}-0.0054 \\
(0.0455)\end{array}$ & $\begin{array}{l}-0.0309 \\
(0.0718)\end{array}$ & $\begin{array}{c}-0.1239 * * \\
(0.0574)\end{array}$ \\
\hline$\kappa$ & $\begin{array}{c}0.0871 \\
(0.0586)\end{array}$ & $\begin{array}{c}0.0587 \\
(0.0712)\end{array}$ & $\begin{array}{c}-0.0238 \\
(0.0959)\end{array}$ & $\begin{array}{c}0.0402 \\
(0.0680)\end{array}$ & $\begin{array}{c}0.0155 \\
(0.0633)\end{array}$ & $\begin{array}{c}0.0749 \\
(0.0555)\end{array}$ \\
\hline \multicolumn{7}{|l|}{ Variance Equation } \\
\hline$\omega$ & $\begin{array}{c}0.2765 \\
(0.1764)\end{array}$ & $\begin{array}{l}0.1619^{* *} \\
(0.0636)\end{array}$ & $\begin{array}{l}-0.1000 * \\
(0.0525)\end{array}$ & $\begin{array}{l}0.0442^{* * *} \\
(0.0171)\end{array}$ & $\begin{array}{c}0.9047^{* * *} \\
(0.0457)\end{array}$ & $\begin{array}{c}1.0285^{* * *} \\
(0.1838)\end{array}$ \\
\hline $\operatorname{ARCH}(\alpha)$ & $\begin{array}{c}0.1750 \\
(0.1256)\end{array}$ & $\begin{array}{c}0.0022 \\
(0.0199)\end{array}$ & $\begin{array}{c}0.0915 \\
(0.0654)\end{array}$ & $\begin{array}{l}-0.0207 \\
(0.0229)\end{array}$ & $\begin{array}{l}0.0910 * * \\
(0.0367)\end{array}$ & $\begin{array}{c}0.0594 \\
(0.0851)\end{array}$ \\
\hline $\operatorname{GARCH}(\beta)$ & $\begin{array}{c}0.5767^{* * *} \\
(0.2034)\end{array}$ & $\begin{array}{c}0.7714^{* * *} \\
(0.0808)\end{array}$ & $\begin{array}{c}0.8115^{* * *} \\
(0.0876)\end{array}$ & $\begin{array}{c}0.9664^{* * *} \\
(0.0273)\end{array}$ & $\begin{array}{c}0.7361^{* * *} \\
(0.1177)\end{array}$ & $\begin{array}{c}0.4306 \\
(0.2625)\end{array}$ \\
\hline$\mu$ & $\begin{array}{c}0.3130 * * \\
(0.1417)\end{array}$ & $\begin{array}{l}0.4084^{* * *} \\
(0.0825)\end{array}$ & $\begin{array}{c}0.3061^{* * *} \\
(0.0965)\end{array}$ & $\begin{array}{c}0.0538^{* * *} \\
(0.0155)\end{array}$ & $\begin{array}{c}0.2760 * * * \\
(0.0561)\end{array}$ & $\begin{array}{c}0.2720^{* * *} \\
(0.0830)\end{array}$ \\
\hline TGARCH $(\gamma)$ & & $\begin{array}{c}0.1343 \\
(0.0874)\end{array}$ & & & & \\
\hline EGARCH $(\delta)$ & & & $\begin{array}{c}-0.1216^{*} \\
(0.0678)\end{array}$ & & & \\
\hline $\operatorname{APARCH}(\gamma)$ & & & & $\begin{array}{l}-0.7531 \\
(1.1979)\end{array}$ & & \\
\hline (A)CGARCH $(\rho)$ & & & & & $\begin{array}{c}0.9192 \\
(0.0102\end{array}$ & $\begin{array}{c}0.9524 * * * \\
(0.0216)\end{array}$ \\
\hline$(\mathrm{A}) \mathrm{CGARCH}(\theta)$ & & & & & $\begin{array}{c}-0.0754^{* * *} \\
(0.0182)\end{array}$ & $\begin{array}{l}-0.0314 \\
(0.0269)\end{array}$ \\
\hline ACGARCH $(\gamma)$ & & & & & & $\begin{array}{c}0.3516^{* *} \\
(0.1474)\end{array}$ \\
\hline $\mathrm{AIC}$ & 2.6401 & 2.7415 & 2.7525 & 2.7384 & 2.6236 & 2.5969 \\
\hline SIC & 2.7575 & 2.8589 & 2.8846 & 2.8558 & 2.7703 & 2.7584 \\
\hline LL & -303.53 & -315.50 & -315.80 & -315.13 & -299.58 & -295.44 \\
\hline JB stat & $\begin{array}{c}456.38 \\
{[0.0000]}\end{array}$ & $\begin{array}{c}180.95 \\
{[0.0000]}\end{array}$ & $\begin{array}{c}296.19 \\
{[0.0000]}\end{array}$ & $\begin{array}{c}233.12 \\
{[0.0000]}\end{array}$ & $\begin{array}{c}141.80 \\
{[0.0000]}\end{array}$ & $\begin{array}{c}135.54 \\
{[0.0000]}\end{array}$ \\
\hline $\mathrm{ARCH}(5)$ & $\begin{array}{c}0.1269 \\
{[0.9862]}\end{array}$ & $\begin{array}{c}0.0971 \\
{[0.9925]}\end{array}$ & $\begin{array}{c}0.1219 \\
{[0.9874]}\end{array}$ & $\begin{array}{c}0.1676 \\
{[0.9743]}\end{array}$ & $\begin{array}{c}0.0445 \\
{[0.9988]}\end{array}$ & $\begin{array}{c}0.1827 \\
{[0.9689]}\end{array}$ \\
\hline$Q^{2}(10)$ & $\begin{array}{c}0.1793 \\
{[0.6720]}\end{array}$ & $\begin{array}{c}0.0284 \\
{[0.8660]}\end{array}$ & $\begin{array}{c}0.0187 \\
{[0.8910]}\end{array}$ & $\begin{array}{c}0.0178 \\
{[0.8940]}\end{array}$ & $\begin{array}{c}0.0015 \\
{[0.9690]}\end{array}$ & $\begin{array}{c}0.2114 \\
{[0.6460]}\end{array}$ \\
\hline
\end{tabular}

Notes: $\left({ }^{*}\right),(* *)$ and $\left({ }^{* * *}\right)$ denote significance at $10 \%, 5 \%$ and $1 \%$, successively. Same notes as in Table A9.

\section{References}

Aalborg, Halvor Aarhus, Peter Molnár, and Jon Erik de Vries. 2019. What can explain the price, volatility and trading volume of bitcoin? Finance Research Letters 29: 255-65. [CrossRef]

Akyildirim, Erdinc, Shaen Corbet, Paraskevi Katsiampa, Neil Kellard, and Ahmet Sensoy. 2019. The development of bitcoin futures: Exploring the interactions between cryptocurrency derivatives. Finance Research Letters. In press.

Ardia, David, Keven Bluteau, and Maxime Rüede. 2019. Regime changes in bitcoin garch volatility dynamics. Finance Research Letters 29: 266-71. [CrossRef]

Bollerslev, Tim. 1986. Generalized autoregressive conditional heteroskedasticity. Journal of Econometrics 31: 307-27. [CrossRef]

Bouoiyour, Jamal, and Refk Selmi. 2015. What does bitcoin look like? Annals of Economics and Finance 16: 449-92. Bouoiyour, Jamal, and Refk Selmi. 2016. Bitcoin: A beginning of a new phase. Economics Bulletin 36: 1430-40. 
Bouri, Elie, Peter Molnár, Georges Azzi, David Roubaud, and Lars Ivar Hagfors. 2017a. On the hedge and safe haven properties of bitcoin: Is it really more than a diversifier? Finance Research Letters 20: 192-98. [CrossRef]

Bouri, Elie, Rangan Gupta, Aviral Kumar Tiwari, and David Roubaud. 2017b. Does bitcoin hedge global uncertainty? Evidence from wavelet-based quantile-in-quantile regressions. Finance Research Letters 23: 87-95. [CrossRef]

Charles, Amélie, and Olivier Darné. 2019. Volatility estimation for bitcoin: Replication and robustness. International Economics 157: 23-32. [CrossRef]

Corbet, Shaen, Brian Lucey, Maurice Peat, and Samuel Vigne. 2018. Bitcoin futures-What use are they? Economics Letters 172: 23-27. [CrossRef]

Dastgir, Shabbir, Ender Demir, Gareth Downing, Giray Gozgor, and Chi Keung Marco Lau. 2019. The causal relationship between bitcoin attention and bitcoin returns: Evidence from the copula-based granger causality test. Finance Research Letters 28: 160-64. [CrossRef]

Demir, Ender, Giray Gozgor, Chi Keung Marco Lau, and Samuel A. Vigne. 2018. Does economic policy uncertainty predict the bitcoin returns? an empirical investigation. Finance Research Letters 26: 145-49. [CrossRef]

Ding, Zhuanxin, Clive W.J. Granger, and Robert F. Engle. 1993. A long memory property of stock market returns and a new model. Journal of Empirical Finance 1: 83-106. [CrossRef]

Dyhrberg, Anne Haubo. 2016a. Bitcoin, gold and the dollar-A garch volatility analysis. Finance Research Letters 16: 85-92. [CrossRef]

Dyhrberg, Anne Haubo. 2016b. Hedging capabilities of bitcoin. Is it the virtual gold? Finance Research Letters 16: 139-44. [CrossRef]

Engle, Robert F., and Gary G.J. Lee. 1993. A Permanent and Transitory Component Model of Stock Return Volatility. Technical report. San Diego: Department of Economics.

Glosten, Lawrence R., Ravi Jagannathan, and David E. Runkle. 1993. On the relation between the expected value and the volatility of the nominal excess return on stocks. The Journal of Finance 48: 1779-1801. [CrossRef]

Hale, Galina, Arvind Krishnamurthy, Marianna Kudlyak, and Patrick Shultz. 2018. How futures trading changed bitcoin prices. FRBSF Economic Letter 12: 1-5.

Katsiampa, Paraskevi. 2017. Volatility estimation for bitcoin: A comparison of garch models. Economics Letters 158: 3-6. [CrossRef]

Katsiampa, Paraskevi. 2018. Volatility co-movement between bitcoin and ether. Finance Research Letters 30: 221-27. [CrossRef]

Katsiampa, Paraskevi. 2019. An empirical investigation of volatility dynamics in the cryptocurrency market. Research in International Business and Finance 50: 322-35. [CrossRef]

Kim, Wonse, Junseok Lee, and Kyungwon Kang. 2019. The effects of the introduction of bitcoin futures on the volatility of bitcoin returns. Finance Research Letters 33: 101204. [CrossRef]

Köchling, Gerrit, Janis Müller, and Peter N. Posch. 2018. Does the introduction of futures improve the efficiency of bitcoin? Finance Research Letters 30: 367-70. [CrossRef]

Kristoufek, Ladislav. 2013. Bitcoin meets google trends and wikipedia: Quantifying the relationship between phenomena of the internet era. Scientific Reports 3: 3415. [CrossRef]

Liu, Ruozhou, Shanfeng Wan, Zili Zhang, and Xuejun Zhao. 2019. Is the introduction of futures responsible for the crash of bitcoin? Finance Research Letters. In press. [CrossRef]

Nakamoto, Satoshi. 2008. Bitcoin: A Peer-to-Peer Electronic Cash System. bitcoin. org. Available online: https:/ / bitcoin.org/bitcoin.pdf (accessed on 21 May 2019).

Nelson, Daniel B. 1991. Conditional heteroskedasticity in asset returns: A new approach. Econometrica: Journal of the Econometric Society 59: 347-70. [CrossRef]

Pal, Debdatta, and Subrata K Mitra. 2019. Hedging bitcoin with other financial assets. Finance Research Letters 30: 30-36. [CrossRef]

Panagiotidis, Theodore, Thanasis Stengos, and Orestis Vravosinos. 2018. On the determinants of bitcoin returns: A lasso approach. Finance Research Letters 27: 235-40. [CrossRef]

Panagiotidis, Theodore, Thanasis Stengos, and Orestis Vravosinos. 2019. The effects of markets, uncertainty and search intensity on bitcoin returns. International Review of Financial Analysis 63: 220-42. [CrossRef]

Panagiotidis, Theodore, Thanasis Stengos, and Orestis Vravosinos. 2020. A principal component-guided sparse regression approach for the determination of bitcoin returns. Journal of Risk and Financial Management 13: 33. [CrossRef] 
Sebastião, Helder, and Pedro Godinho. 2019. Bitcoin futures: An effective tool for hedging cryptocurrencies. Finance Research Letters 33: 101230. [CrossRef]

Stein, Jeremy C. 1987. Informational externalities and welfare-reducing speculation. Journal of Political Economy 95: 1123-45. [CrossRef]

Tay, J. Kenneth, Jerome Friedman, and Robert Tibshirani. 2018. Principal component-guided sparse regression. arXiv arXiv:1810.04651.

Wu, Shan, Mu Tong, Zhongyi Yang, and Abdelkader Derbali. 2019. Does gold or bitcoin hedge economic policy uncertainty? Finance Research Letters 31: 171-78. [CrossRef]

Yermack, David. 2015. Is bitcoin a real currency? An economic appraisal. In Handbook of Digital Currency. Amsterdam: Elsevier, pp. 31-43.

(C) 2020 by the authors. Licensee MDPI, Basel, Switzerland. This article is an open access article distributed under the terms and conditions of the Creative Commons Attribution (CC BY) license (http://creativecommons.org/licenses/by/4.0/). 